\title{
Novel Clustering Schemes for Full and Compact Polarimetric SAR Data: An Application for Rice Phenology Characterization
}

\author{
Subhadip Dey ${ }^{a, *}$, Avik Bhattacharya ${ }^{a}$, Debanshu Ratha ${ }^{\mathrm{a}}$, \\ Dipankar Mandal ${ }^{a}$, Heather McNairn ${ }^{b}$, Juan M. Lopez-Sanchez ${ }^{c}$, Y. S. Rao ${ }^{a}$ \\ ${ }^{a}$ Microwave Remote Sensing Lab, CSRE, Indian Institute of Technology Bombay, \\ Mumbai, India \\ ${ }^{b}$ Ottawa Research and Development Centre, Agriculture and Agri-Food Canada, Canada \\ ${ }^{c}$ University of Alicante, Alicante, Spain
}

\section{Abstract}

Information on rice phenological stages from Synthetic Aperture Radar (SAR) images is of prime interest for in-season monitoring. Often, prior in-situ measurements of phenology are not available. In such situations, unsupervised clustering of SAR images might help in discriminating phenological stages of a crop throughout its growing period. Among the existing unsupervised clustering techniques using full-polarimetric (FP) SAR images, the eigenvalue-eigenvector based roll-invariant scattering-type parameter, and the scattering entropy parameter are widely used in the literature. In this study, we utilize a unique target scattering-type parameter, which jointly uses the Barakat degree of polarization and the elements of the polarimetric coherency matrix. Likewise, we also utilize an equivalent parameter proposed for compact-polarimetric (CP) SAR data. These scattering-type parameters are analogous to the Cloude-Pottier's parameter for FP SAR data and the

\footnotetext{
*Corresponding author: Subhadip Dey (sdey2307@gmail.com)
} 
ellipticity parameter for CP SAR data. Besides this, we also introduce new clustering schemes for both FP and CP SAR data for segmenting diverse scattering mechanisms across the phenological stages of rice. In this study, we use the RADARSAT-2 FP and simulated CP SAR data acquired over the Indian test site of Vijayawada under the Joint Experiment for Crop Assessment and Monitoring (JECAM) initiative. The temporal analysis of the scattering-type parameters and the new clustering schemes help us to investigate detailed scattering characteristics from rice across its phenological stages.

Keywords: Unsupervised clustering, Entropy, RADARSAT-2, Crop monitoring, PolSAR, Roll-invariant parameter

1. Introduction

2 Variations in crop phenological stages can be characterized by Synthetic 3 Aperture Radar (SAR) data due to its high sensitivity to the dielectric and 4 geometrical structure of the canopy. However, depending on the frequency 5 of the transmitted electromagnetic (EM) wave, the interaction with crop 6 canopy layers and the underlying soil varies significantly (Davidson et al., 2000). Previous studies reported that phenological changes could be ade8 quately captured with high-frequency SAR sensors utilizing backscattered 9 information from vegetation canopy (Wiseman et al., 2014; De Bernardis et al., 2015; McNairn and Shang, 2016; McNairn et al., 2018). In general, the SAR backscatter signal might be affected by the underlying surface during early vegetative growth stages when the canopy was sparse and open (Paloscia, 2002). 
One of the primary parameters associated with the changes in the SAR backscatter coefficient is the crop canopy distribution (e.g., tillers, leaves, and panicles) at each phenological stage. Moreover, this distribution in the crop fields also leads to randomness in scattering (Yuzugullu et al., 2015). In such situations, polarimetric entropy $(H)$ is an important parameter to quantify this randomness. In Cloude and Pottier (1997), an unsupervised classification scheme $(H / \bar{\alpha})$ was proposed using $H$ and the average scattering-type parameter $(\bar{\alpha})$. The $H / \bar{\alpha}$ plane is sub-divided into nine zones to suitably cluster various scattering mechanisms. The properties of different scattering mechanisms determine the boundaries between the zones. Hence certain assumptions are utilized in the proper setting of these boundaries. Subsequently, the $2 \mathrm{D}$ clustering plane is extended to $3 \mathrm{D} H / A / \bar{\alpha}$ space by introducing the scattering anisotropy parameter $A$. This parameter, which is complementary to $H$, is useful to discriminate targets when $H>0.7$. However, for lower values of $H$, this parameter is noisy and could introduce inaccuracies in determining the clusters.

Lopez-Sanchez et al. (2011) reported the importance of the $H / \bar{\alpha}$ plane to discriminate phenological stages of rice along with the temporal correlation of $\mathrm{HH}$ and $\mathrm{VV}$ and their ratio. The clustering results show that at the beginning of the cultivation period of rice, the data cluster was denser in the region with medium entropy and low alpha, which was primarily due to the presence of sparse vegetation in the fields. However, at the advanced phenological stages, the cluster density shifted towards the region of high entropy and high alpha in the $H / \bar{\alpha}$ plane.

In another study, Lopez-Sanchez et al. (2012) utilized the dominant scattering- 
type information $\left(\alpha_{1}\right)$ instead of $\bar{\alpha}$. In this study, the temporal behaviour of $\alpha_{1}$ and the scattering entropy was shown with the phenological stages of rice. At the initial stage, $\alpha_{1}$ and entropy were both within low to medium values, and they jointly increased during the plant emergence stage. During the advanced vegetative stage, both parameters show the dominance of multiple scattering from the fields. In contrast, at the harvest stage, $\alpha_{1}<30^{\circ}$ and the scattering entropy remained high due to the field roughness condition.

Praks et al. (2009) proposed alternative scattering-type and randomness parameters equivalent to $\bar{\alpha}$ and $H$ for clustering PolSAR data. These parameters can be directly obtained from the elements of the coherency matrix without utilizing the eigenvalues and the eigenvectors. It was shown that instead of $\bar{\alpha}$ and $H$, the surface scattering fraction and the scattering diversity that are equivalent polarimetric descriptors can be utilized for classification, visualization, or interpretation. Later, Yin et al. (2015) proposed a new parameter, $\alpha_{B}$, defined by the co-polarization ratio and their coherence to capture various scattering mechanisms. This new parameter was able to distinguish scattering from oriented and randomly distributed targets. In their study a new $\Delta \alpha_{\mathrm{B}} / \alpha_{\mathrm{B}}$ plane was proposed which showed better separation capability than the $H / \bar{\alpha}$ clustering plane. It was also stated that the stability of the proposed method was better with multi-temporal SAR data.

In another work, Ratha et al. (2019) proposed a roll-invariant scatteringtype parameter $\left(\alpha_{G D}\right)$, the helicity parameter $\left(\tau_{G D}\right)$, and the purity parameter $\left(P_{G D}\right)$ using a geodesic distance between two Kennaugh matrices. A new $P_{G D} / \alpha_{G D}$ unsupervised classification scheme is proposed which is analogous to $H / \bar{\alpha}$. However, the $P_{G D} / \alpha_{G D}$ clustering plane showed better performance 
than earlier proposed schemes.

The study using compact-polarimetric (CP) SAR data holds promise due to the upcoming constellation of satellites such as the Canadian RADARSAT Constellation Mission (RCM), SAOCOM (TOPSAR with experimental CP-mode), and the NISAR (the NASA-ISRO SAR) L- and S-band mission. Similar to the full-polarimetric (FP) case, scattering-type clustering assessment using compact polarimetric (CP) SAR data and its decomposition parameters (Raney, 2007; Cloude et al., 2011; Raney et al., 2012) are lately gaining interest (Ainsworth et al., 2009; Charbonneau et al., 2010; BallesterBerman and Lopez-Sanchez, 2011; Sabry and Vachon, 2013). Brisco et al. (2013) assessed hybrid-compact, circular, and linear polarimetric SAR data for rice and wetlands mapping. Also, different dual-channel combinations and $m-\delta$ decomposition parameters for CP data were assessed in their study, where the classification accuracy for CP data was comparatively better than linear dual-polarimetric SAR data.

Lopez-Sanchez et al. (2014) used the radar backscatter coefficients and the $H / \bar{\alpha}$ plane to investigate the dynamics of rice phenological changes for full, dual, and compact polarimetric SAR data. In this study, the dominant scattering-type parameter $\left(\alpha_{s}\right)$ for CP data is used instead of $\bar{\alpha}$. For CP data, the entropy, in particular, is equivalent to the Barakat degree of polarization. It was noticed that the pattern of $\alpha_{s}$ was similar for full, dual, and compact polarimetric SAR data for rice crops. Alongside this, it was also observed that $\alpha_{s}$ precisely provides similar information like the FP mode, throughout the phenological cycle of rice.

Subsequently, Yang et al. (2014) showed improved classification accuracy 
in discriminating transplanted and direct-sown rice fields. In this study, the use of the $m-\chi$ decomposition parameters along with $\alpha_{s}$, the degree of polarization $(m)$, relative phase $(\delta)$ and conformity coefficient $(\mu)$ improved the classification accuracy from $88 \%$ to $95 \%$. Besides, the classification accuracy confirmed the advantage of CP data over other dual-polarized SAR data. Several other studies (Xie et al., 2015; Uppala et al., 2015; Guo et al., 2018; Kumar et al., 2020) also indicated the potential of CP SAR data for rice mapping and monitoring.

Recently, Yin et al. (2019) proposed a new parameter, $\alpha_{B C P}$, for improvement in the clustering results for land-cover features. In particular, $\alpha_{B C P}$ is rotation-invariant and $\Delta \alpha_{B C P} / \alpha_{B C P}$ resembles the existing $\Delta \alpha_{B} / \alpha_{B}$ clustering for FP SAR data. However, the differences between $\alpha_{B C P}$ and $\alpha_{B}$ depend on the polarization of the received wave. Moreover, the derivation of specific scattering models is needless for separate CP modes. It was also observed that circular CP data provides almost similar results as FP data for various scattering targets.

The literature, as mentioned above, provides a vital foundation for the utilization of $H$ and the scattering-type parameters (i.e., $\bar{\alpha}$ and $\alpha_{s}$ ) for rice crop monitoring and mapping using FP and CP SAR data. Nevertheless, these techniques are formulated either by fitting scattering models or by diagonalizing the coherency (or covariance) matrix of the received wave. Hence, these techniques might miss the received antenna basis invariant information while characterizing various targets. The importance of the received antenna basis invariant information in terms of the degree of polarization helps to effectively exploit complete information from SAR data (Touzi et al., 2015, 
2018).

In this study, our main objective is to characterize changes in scattering mechanisms utilizing the temporal series of full- and compact polarimetric SAR data across the growth stages of rice. The received antenna basis invariant information, i.e., in particular, the Barakat degree of polarization (Barakat, 1977, 1983) is useful to capture changes in scattering randomness due to crop foliage development. At the same time, the elements of the coherency (or, covariance) matrices provide information about crop canopy geometry as well as the soil and vegetation water content. In this regard, a new scattering-type parameter is derived by jointly using the received antenna basis invariant information and elements of coherency (or, covariance) matrix for both FP and CP SAR data. Alongside this, we present a comparative study of the performance of novel clustering schemes for FP and $\mathrm{CP}$ data for rice phenology mapping. It is noteworthy that the formulation of this new scattering-type parameter is equivalent for both FP and CP SAR data.

Here, we have proposed new clustering schemes using $\theta_{\mathrm{FP}}$ and $\theta_{\mathrm{CP}}$ along with $H$ for both FP and CP SAR data, respectively. Unlike the $H / \alpha$ plane, the proposed segmentation scheme utilizes a polar representation, which offers a natural choice. Suitable entropy apportionment (radially) together with angular extent of $\theta_{X} \in\left[-90^{\circ}, 90^{\circ}\right]$ (where the subscript $X$ is either FP or CP) provides a reliable target discrimination strategy. The segmentation scheme produces 12 feasible clustering zones that better characterize natural and human-made targets. The usefulness and performance of the scatteringtype parameters $\theta_{\mathrm{FP}}$ and $\theta_{\mathrm{CP}}$, along with the new clustering schemes, are 
assessed by utilizing them with the time-series C-band RADARSAT-2 data for monitoring rice.

\section{Study area and field measurement}

The study area is located near Vijayawada in the state of Andhra Pradesh, India $\left(16^{\circ} 24^{\prime} 6.2^{\prime \prime} N, 8^{\circ} 41^{\prime} 2.4^{\prime \prime} E\right)$ as shown in figure 1 (Mandal et al., 2019). The climatic zone of this area varies from sub-humid to humid, with mostly clayey soil texture. Areal coverage of this test site is $\approx 25 \times 25 \mathrm{~km}^{2}$. Rice is one of the primary and major crops cultivated in this area. The sowing period of rice varies from mid of June to mid of July depending on the variety and cultivation practices. Majorly, the cultivation starts after the pre-monsoon rain and is harvested during mid-December. The average size of each field was $\approx 60 \times 60 \mathrm{~m}^{2}$, and in each field, two sampling locations were chosen for in-situ measurements. Information about the crop growth stages, management practices, and biophysical parameters was noted during the field campaign from June to December 2018.

Table 1: Statistics (mean \pm standard deviation) of bio-physical and soil parameters at different phenology stages of rice. Here, PH: plant height, PAI: plant area index, SM: soil moisture and Nan: Not a number

\begin{tabular}{ccccl}
\hline Date & PH $(\mathrm{cm})$ & PAI $\left(\mathrm{m}^{2} \mathrm{~m}^{-2}\right)$ & $\mathbf{S M}(\%)$ & Growth stage \\
\hline $05 / 07 / 2018$ & Nan & Nan & $35.92 \pm 6.6$ & Bare field \\
\hline $29 / 07 / 2018$ & $26.30 \pm 5.21$ & $0.40 \pm 0.20$ & Saturated & Early tillering \\
\hline $22 / 08 / 2018$ & $46.26 \pm 9.12$ & $1.76 \pm 0.26$ & Saturated & $\begin{array}{l}\text { Advanced } \\
\text { tillering }\end{array}$ \\
\hline $09 / 10 / 2018$ & $92.16 \pm 5.76$ & $4.03 \pm 0.20$ & Saturated & Flowering \\
\hline $02 / 11 / 2018$ & $95.93 \pm 7.76$ & $4.06 \pm 0.16$ & $47.60 \pm 0.42$ & Early dough \\
\hline $26 / 11 / 2018$ & $98.32 \pm 6.82$ & $3.86 \pm 0.22$ & $45.16 \pm 6.04$ & Maturity \\
\hline
\end{tabular}


A total number of 14 in-situ field measurements were considered in this study. We measured soil moisture at each field in two sampling locations, arranged in two parallel transects along the row direction. The separation between each transect was $\approx 40 \mathrm{~m}$. We measured the pointwise soil moisture using theta-probe. Nevertheless, the soil underlying the rice crops was saturated during the majority of the growth stages due to irrigation and rainfall events. We measured vegetation samples at two points of each field due to the spatial heterogeneity within the field, which is due to the irregular growth pattern of rice. Vegetation sampling included the measurement of PAI, plant height, and phenology through non-destructive methods. The PAI is measured using the notion of hemispherical digital photography. During each measurement day, we took ten photos along two transects which are separated by $2 \mathrm{~m}$ in each sampling point, using a wide-angle lens mounted on a digital camera. All images were post-processed using the CanEYE software to provide an estimate of PAI. We have sampled the vegetation crop water content intermittently at few phenological stages. At the maturity stage, the water content in the grain was $14.2 \%$ to $19.6 \%$ (wet basis) while the stem water content got reduced by $36 \%$ to $42 \%$ (wet basis) as compared to the dough stage. The overall phenology of rice is usually expressed with three major stages: vegetative, reproductive, and mature (or ripening). The statistics of bio-physical and soil parameters are given in Table 1.

\section{Satellite data pre-processing}

We acquired RADARSAT-2 images in Fine Quad (FQ) wide mode from July to November 2018 over the test site as shown in Table 2. We then 


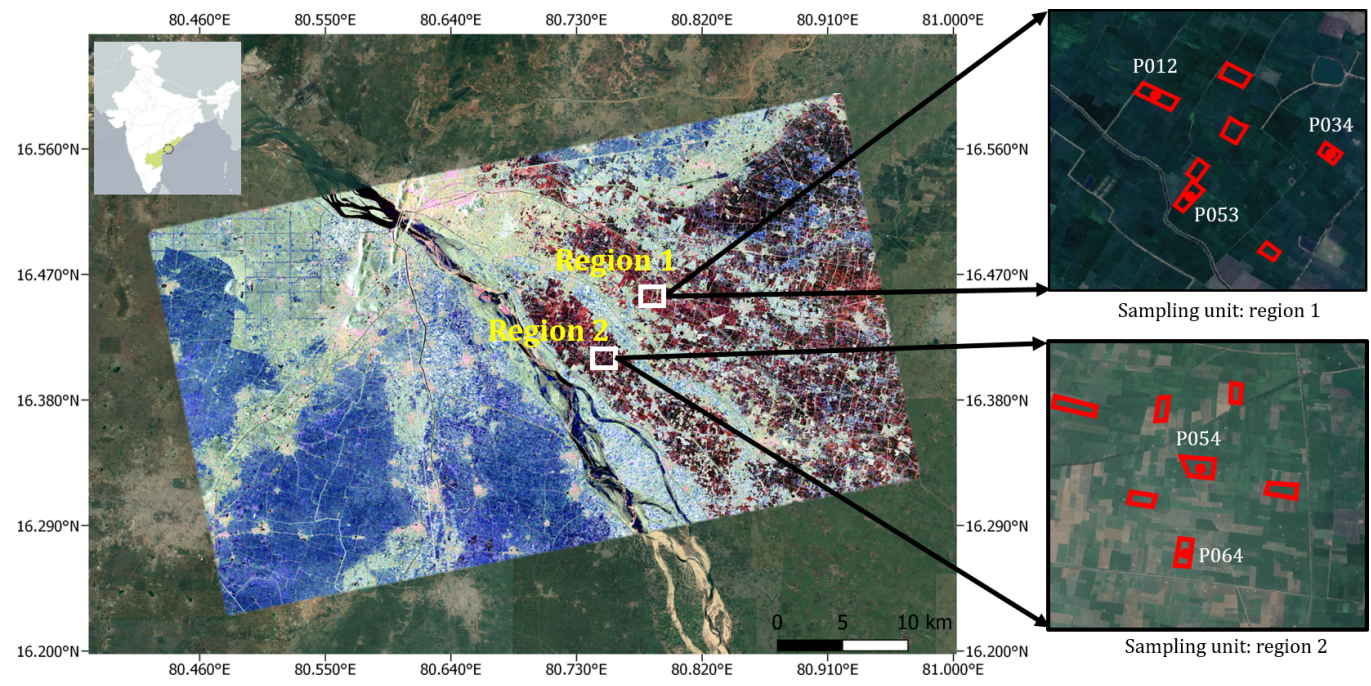

Figure 1: The Google Earth image of the JECAM test site over Vijayawada, India is overlaid with a Pauli RGB image obtained from SAR data acquired on 29 Jul 2018. The samples from region 1 and 2 are used for temporal analysis and clustering. The distribution of five in-situ data points is shown in the sampling unit of region 1 and region 2 .

apply a multi-look factor of $2 \times 3$ pixels in the range and azimuth directions, respectively, to generate $\approx 15 \mathrm{~m}$ square pixel images. In general, the parcel sizes in this test area are small. However, during rice cultivation, many fields are cultivated alongside the field boundaries. Therefore, the fields seem to be quasi-homogeneous, depending on cultivation practices. Since the area is quasi-homogeneous, we apply a $3 \times 3$ boxcar filter (Lee and Pottier, 2009) to each coherency matrix $(\mathbf{T})$ in the images for speckle reduction. Furthermore, we generate simulated compact polarimetric (CP) SAR data from the FP data with $0^{\circ}$ orientation angle and $-45^{\circ}$ ellipticity angle (shown in Appendix B). We co-register all FP and CP images with the RMSE $\leq 0.25 \mathrm{~m}$. 
Table 2: Specification of the C-band full-pol RADARSAT-2 acquisitions over the test site during the field campaign ( $a z$ : azimuth resolution and $r g$ : range resolution)

\begin{tabular}{ccccc}
\hline $\begin{array}{c}\text { Acquisition } \\
\text { date }\end{array}$ & $\begin{array}{c}\text { Beam } \\
\text { mode }\end{array}$ & $\begin{array}{c}\text { Incidence angle } \\
\text { range (deg.) }\end{array}$ & Orbit & $a z(\mathrm{~m}) \times r g(\mathrm{~m})$ \\
\hline $05 / 07 / 2018$ & FQ15W & $33.73-36.65$ & Ascending & $4.73 \times 5.11$ \\
$29 / 07 / 2018$ & FQ15W & $33.73-36.65$ & Ascending & $4.73 \times 5.11$ \\
$22 / 08 / 2018$ & FQ15W & $33.73-36.65$ & Ascending & $4.73 \times 5.11$ \\
$09 / 10 / 2018$ & FQ15W & $33.73-36.65$ & Ascending & $4.73 \times 5.11$ \\
$02 / 11 / 2018$ & FQ15W & $33.73-36.64$ & Ascending & $4.73 \times 5.11$ \\
$26 / 11 / 2018$ & FQ15W & $33.73-36.64$ & Ascending & $4.73 \times 5.11$ \\
\hline
\end{tabular}

\section{Methodology}

In this section, we present the newly proposed scattering-type parameters for both full- and compact-pol SAR data (Dey et al., 2020) for monitoring rice crop. Alongside this, we propose an unsupervised clustering scheme utilizing these new parameters along with the scattering entropy parameter (i.e., a measure of randomness) derived from full (FP) and compact-pol (CP)

SAR data.

\subsection{Full-polarimetry}

In FP SAR, the $2 \times 2$ complex scattering matrix $\mathbf{S}$ encompasses complete polarimetric information about backscattering from targets for each pixel. It is expressed in the backscatter alignment (BSA) convention in the linear horizontal $(\mathrm{H})$ and linear vertical $(\mathrm{V})$ polarization basis as,

$$
\mathbf{S}=\left[\begin{array}{ll}
S_{\mathrm{HH}} & S_{\mathrm{HV}} \\
S_{\mathrm{VH}} & S_{\mathrm{VV}}
\end{array}\right]
$$


Each element of the matrix represents the backscattering response of the target at a specific polarization. The diagonal elements of the matrix represent the co-polarized scattering information, while the off-diagonal terms represent the cross-pol information. In the monostatic backscattering case, the reciprocity theorem constrains the scattering matrix to be symmetric, i.e., $S_{\mathrm{HV}}=S_{\mathrm{VH}}$.

To reduce the speckle effect in $\mathbf{S}$, the multi-looked Hermitian positive semi-definite $3 \times 3$ coherency matrix $\mathbf{T}$ is obtained from the averaged outer product of the target vector $\boldsymbol{k}_{P}$ (derived using the Pauli basis matrix, $\Psi_{P}$ ) with its conjugate (Lee and Pottier, 2009).

$$
\begin{gathered}
\Psi_{P}=\left\{\sqrt{2}\left[\begin{array}{ll}
1 & 0 \\
0 & 1
\end{array}\right], \sqrt{2}\left[\begin{array}{cc}
1 & 0 \\
0 & -1
\end{array}\right], \sqrt{2}\left[\begin{array}{ll}
0 & 1 \\
1 & 0
\end{array}\right]\right\} \\
\boldsymbol{k}_{P}=\frac{1}{2} \operatorname{Tr}\left(\mathbf{S} \Psi_{P}\right) \Longrightarrow \boldsymbol{k}_{P}=\frac{1}{\sqrt{2}}\left[S_{H H}+S_{V V}, S_{H H}-S_{V V}, 2 S_{H V}\right]^{T} \\
\mathbf{T}=\frac{1}{N} \sum_{i=1}^{N} \boldsymbol{k}_{P i} \boldsymbol{k}_{P i}^{* T}
\end{gathered}
$$

where $N$ denotes the square window size for spatial averaging and $\operatorname{Tr}$ is the sum of the diagonal elements of the matrix.

When a polarized electromagnetic (EM) wave scatters from a random mixture of targets, it becomes partially polarized. The state of polarization of a partially polarized EM wave is characterized in terms of the degree of polarization $(0 \leq m \leq 1)$. The degree of polarization is defined as the ratio of the (average) intensity of the polarized portion of the wave to that of the (average) total intensity of the wave. For a completely polarized EM wave, 
$m=1$ and for a completely unpolarized EM wave, $m=0$. In between these two extreme cases, the EM wave is said to be partially polarized, $0<m<1$. Barakat (Barakat, 1977) provided an expression of $m$ for the $N \times N$ coherency matrix. This expression is used in this study to obtain the degree of polarization $m_{\mathrm{FP}}$ from the $3 \times 3$ coherency matrix $\mathbf{T}$ for FP SAR data as,

$$
m_{\mathrm{FP}}=\sqrt{1-\frac{27|\mathbf{T}|}{(\operatorname{Tr}(\mathbf{T}))^{3}}},
$$

where $|\cdot|$ is the determinant of a matrix.

From the interpretation of the Huynen parameters in terms of certain general properties of the target geometry, it can be inferred that $T_{11}$ is the generator of target symmetry and represents the scattered power from a regular, smooth and convex parts of the scatterer. Similarly, $\left(T_{22}+T_{33}\right)$ is the generator of the target structure and represents the scattered power from an irregular, uneven and non-convex parts of the scatterer (Lee and Pottier, 2009). Therefore, with respect to the total polarized scattered power (i.e., $m_{\mathrm{FP}}$ Span) from a scatterer, let us denote,

$$
\tan \eta_{1}=\frac{T_{11}}{m_{\mathrm{FP}} \mathrm{Span}} \quad \text { and } \quad \tan \eta_{2}=\frac{T_{22}+T_{33}}{m_{\mathrm{FP}} \mathrm{Span}}
$$

where, $T_{11}=\left\langle\left|S_{\mathrm{HH}}+S_{\mathrm{VV}}\right|^{2}\right\rangle, T_{22}=\left\langle\left|S_{\mathrm{HH}}-S_{\mathrm{VV}}\right|^{2}\right\rangle$, and $T_{33}=4\left\langle\left|S_{\mathrm{HV}}\right|^{2}\right\rangle$ are the diagonal elements of the $\mathbf{T}$ matrix. The total power, Span is defined in terms of the elements of the $\mathbf{T}$ matrix as,

$$
\text { Span }=T_{11}+T_{22}+T_{33} .
$$


Here, $\eta_{1}$ and $\eta_{2}$ are two auxiliary variables representing the tangent of the ratios between the diagonal elements $\left(T_{11}\right.$ and $\left.T_{22}+T_{33}\right)$ of the coherency matrix, $\mathbf{T}$, and the total polarized scattering power ( $\left.m_{\mathrm{FP}} \mathrm{Span}\right)$.

We define:

$$
\tan \gamma_{\mathrm{FP}}=\tan \left(\eta_{1}-\eta_{2}\right)
$$

where $\gamma_{\mathrm{FP}}$ can be related to the average scattering-type parameter, Cloude $\bar{\alpha} \in\left[0^{\circ}, 90^{\circ}\right]$ (Cloude and Pottier, 1997). However, in order to compare the two parameters within the same range, they are suitably modified as, $\widehat{\alpha}=90^{\circ}-2 \bar{\alpha}$ and $\theta_{\mathrm{FP}}=2 \gamma_{\mathrm{FP}}$, which is a roll-invariant parameter (detailed in Appendix A.1) is given as,

$$
\theta_{\mathrm{FP}}=2 \tan ^{-1}\left(\frac{m_{\mathrm{FP}} \operatorname{Span}\left(T_{11}-T_{22}-T_{33}\right)}{T_{11}\left(T_{22}+T_{33}\right)+m_{\mathrm{FP}}^{2} \operatorname{Span}^{2}}\right) \in\left[-90^{\circ}, 90^{\circ}\right] .
$$

40 random (or depolarized).

The eigen-decomposition of $\mathbf{T}$ can be expressed as,

$$
\mathbf{T}=\mathbf{U}_{3} \Sigma \mathbf{U}_{3}^{-1}
$$

where $\boldsymbol{\Sigma}$ is the $3 \times 3$ diagonal matrix with non-negative elements, $\lambda_{1} \geq \lambda_{2} \geq$ $\lambda_{3} \geq 0$, which are the eigenvalues of $\mathbf{T}$. The pseudo probabilities, $p_{i}$ obtained 
248

from the eigenvalues are defined as,

$$
p_{i}=\frac{\lambda_{i}}{\sum_{k=1}^{3} \lambda_{k}},
$$

249

250

which are then used to define the scattering entropy (Lee and Pottier, 2009) as,

$$
H_{\mathrm{FP}}=-\sum_{k=1}^{3} p_{k} \log _{3}\left(p_{k}\right),
$$

251 However, in this study, we use the quantity $\bar{H}_{\mathrm{FP}}=1-H_{\mathrm{FP}}$ in the $\bar{H}_{\mathrm{FP}} / \theta_{\mathrm{FP}}$ polar plot as shown in figure 2 . The feasible regions in the $\bar{H}_{\mathrm{FP}} / \theta_{\mathrm{FP}}$ polar

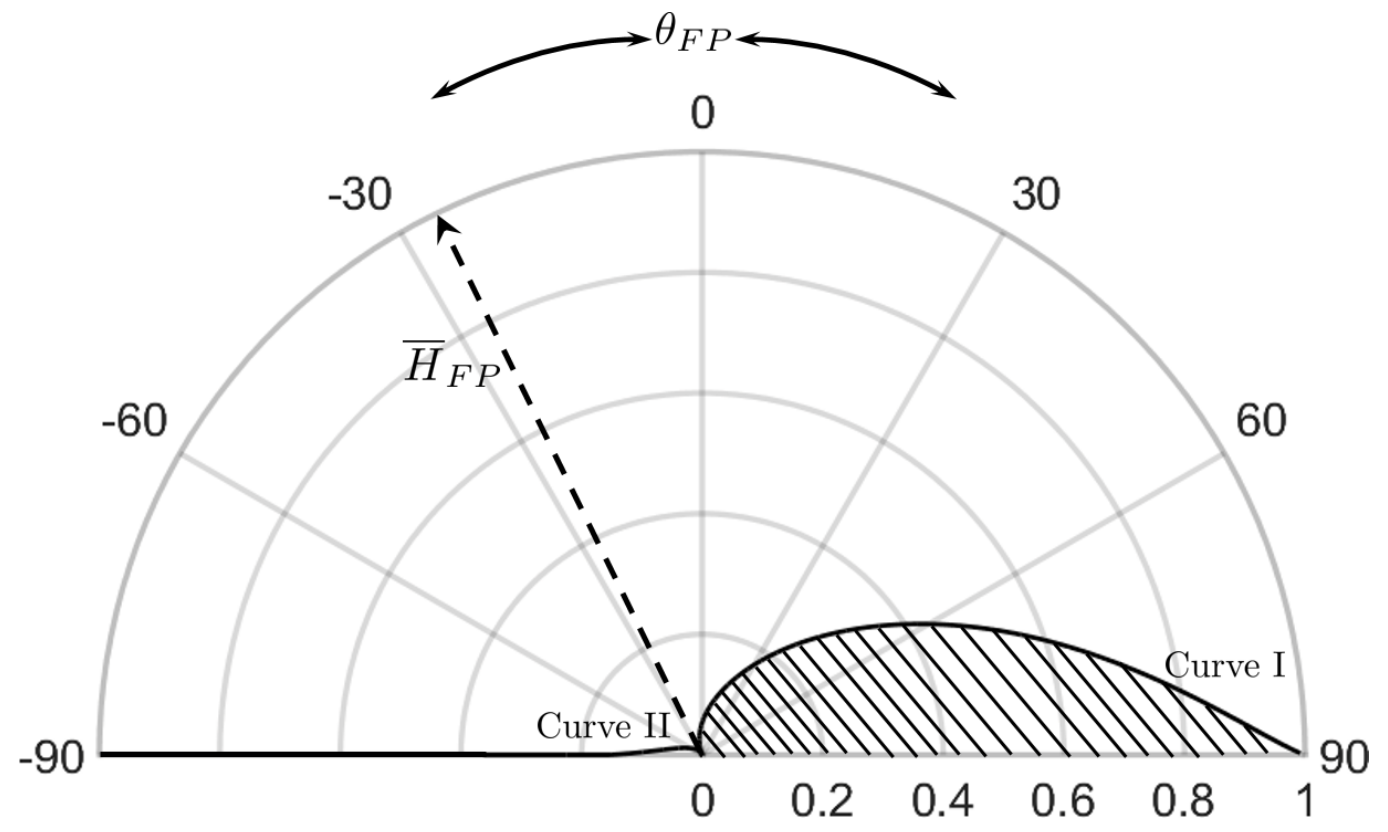

Figure 2: The $\bar{H}_{\mathrm{FP}} / \theta_{\mathrm{FP}}$ plane displayed in polar plot. Curve I and Curve II represent the azimuthal symmetry lines. No scattering mechanisms exist in the shaded portion of the plane. 
253

plot is represented by two bounding curves, Curve I and Curve II in figure 2.

$$
\begin{aligned}
& \text { Curve I, }[\mathbf{T}]_{I}=\left[\begin{array}{lll}
1 & 0 & 0 \\
0 & m & 0 \\
0 & 0 & m
\end{array}\right] \quad 0 \leq m \leq 1 \\
& \text { Curve II, }[\mathbf{T}]_{I I}=\left[\begin{array}{rrrr}
2 m-1 & 0 & 0 \\
0 & 1 & 0 \\
0 & 0 & 1
\end{array}\right] \quad 0.5 \leq m \leq 1
\end{aligned}
$$

254

255 256

\subsection{Compact-polarimetry}

The CP mode measures a projection of the $2 \times 2$ complex scattering matrix $\mathbf{S}$ as,

$$
\begin{aligned}
{\left[\begin{array}{c}
E_{C H} \\
E_{C V}
\end{array}\right] } & =\frac{1}{\sqrt{2}}\left[\begin{array}{ll}
S_{H H} & S_{H V} \\
S_{V H} & S_{V V}
\end{array}\right]\left[\begin{array}{c}
1 \\
\pm i
\end{array}\right] \\
& =\frac{1}{\sqrt{2}}\left[\begin{array}{c}
S_{H H} \pm i S_{H V} \\
S_{V H} \pm i S_{V V}
\end{array}\right]
\end{aligned}
$$

where the subscript $C$ can be either the left-hand circular (L) transmit with $a+\operatorname{sign}$ or the right-hand circular $(R)$ transmit with a $-\operatorname{sign}$. The $2 \times 2$ covariance matrix is then obtained from the elements of the scattering vector as,

$$
\mathbf{C}_{2}=\left[\begin{array}{cc}
\left\langle\left|E_{C H}\right|^{2}\right\rangle & \left\langle E_{C H} E_{C V}^{*}\right\rangle \\
\left\langle E_{C V} E_{C H}^{*}\right\rangle & \left\langle\left|E_{C V}\right|^{2}\right\rangle
\end{array}\right]
$$

261 For CP-SAR data, the $4 \times 1$ Stokes vector $\vec{g}$ can be written in terms of 
the elements of the $2 \times 2$ covariance matrix $\mathbf{C}_{2}$ as,

$$
\overrightarrow{\mathbf{g}}=\left[\begin{array}{c}
g_{0} \\
g_{1} \\
g_{2} \\
g_{3}
\end{array}\right]=\left[\begin{array}{c}
C_{11}+C_{22} \\
C_{11}-C_{22} \\
C_{12}+C_{21} \\
\pm j\left(C_{12}-C_{21}\right)
\end{array}\right]
$$

where \pm corresponds to left and right circular polarization respectively.

From the elements of $\vec{g}$, the backscatter power in the same sense $(S C=$ $\left.\frac{g_{0}-g_{3}}{2}\right)$ and opposite sense $\left(O C=\frac{g_{0}+g_{3}}{2}\right)$ to the transmitted circular polarization is utilized to derive the scattering-type parameter for the compactpolarimetric SAR data similar to the FP case. Here, $O C$ is the generator of target symmetry and represents the scattered power from a regular, smooth and convex parts of the scatterer. Similarly, $S C$ is the generator of the target structure and represents the scattered power from an irregular, uneven and non-convex parts of the scatterer:

$$
\tan \zeta_{1}=\frac{O C}{m_{\mathrm{CP}} \operatorname{Span}}, \quad \text { and } \tan \zeta_{2}=\frac{S C}{m_{\mathrm{CP}} \operatorname{Span}}
$$

where the total power Span is defined as,

$$
\text { Span }=S C+O C
$$

Here, $\zeta_{1}$ and $\zeta_{2}$ are two auxiliary variables representing the tangent of the ratios between the opposite and same sense circular polarized backscatter powers $(O C$ and $S C)$ and the total polarized scattering power $\left(m_{\mathrm{CP}} \mathrm{Span}\right)$. 
Similar to FP, we define:

$$
\tan \gamma_{\mathrm{CP}}=\tan \left(\zeta_{1}-\zeta_{2}\right)
$$

where $\gamma_{\mathrm{CP}}$ can be analogously related to the polarization ellipticity parameter $\chi \in\left[-45^{\circ}, 45^{\circ}\right]$. However, in order to compare, the two parameters within the same range, they are suitably scaled as, $\bar{\chi}=-2 \chi$ and $\theta_{\mathrm{CP}}=2 \gamma_{\mathrm{CP}}$ which is a roll-invariant parameter (detailed in Appendix A.2) is given as,

$$
\theta_{\mathrm{CP}}=2 \tan ^{-1}\left(\frac{m_{\mathrm{CP}} \operatorname{Span}(O C-S C)}{O C \times S C+m_{\mathrm{CP}}^{2} \mathrm{Span}^{2}}\right) \in\left[-90^{\circ}, 90^{\circ}\right]
$$

Similar to $\theta_{\mathrm{FP}}$, it can be noticed from (18) that for a pure dihedral scatterer, i.e., when $O C=0$ and $m_{\mathrm{CP}}=1$, then $\mathrm{Span}=S C$ and $\theta_{\mathrm{CP}}=-90^{\circ}$. Similarly, for a pure trihedral scatterer, i.e., when $S C=0$ and $m_{\mathrm{CP}}=1$, then Span $=O C$ and $\theta_{\mathrm{CP}}=90^{\circ}$. Besides, as $\theta_{\mathrm{CP}}$ approaches 0 , scattering randomness increases and at $\theta_{\mathrm{CP}}=0^{\circ}$, the scattering is purely random (or depolarized).

The expression for the Barakat degree of polarization for the compactpolarimetric case is given as,

$$
m_{\mathrm{CP}}=\sqrt{1-\frac{4\left|\mathbf{C}_{2}\right|}{\left(\operatorname{Tr}\left(\mathbf{C}_{2}\right)\right)^{2}}}
$$

The eigen-decomposition of $\mathbf{C}_{2}$ can be expressed as,

$$
\mathbf{C}_{2}=\mathbf{U}_{2} \Sigma \mathbf{U}_{2}^{-1}
$$




$$
p_{i}=\frac{\lambda_{i}}{\sum_{k=1}^{2} \lambda_{k}},
$$

which are then used to define the scattering entropy $\left(H_{\mathrm{CP}}\right)$ for CP-SAR data as,

$$
H_{\mathrm{CP}}=-\sum_{k=1}^{2} p_{k} \log _{2}\left(p_{k}\right) .
$$

As mentioned earlier for the FP case, we use the quantity $\bar{H}_{\mathrm{CP}}=1-H_{\mathrm{CP}}$ in the $\bar{H}_{\mathrm{CP}} / \theta_{\mathrm{CP}}$ polar plot as shown in figure 3 . Similar to FP, the feasible

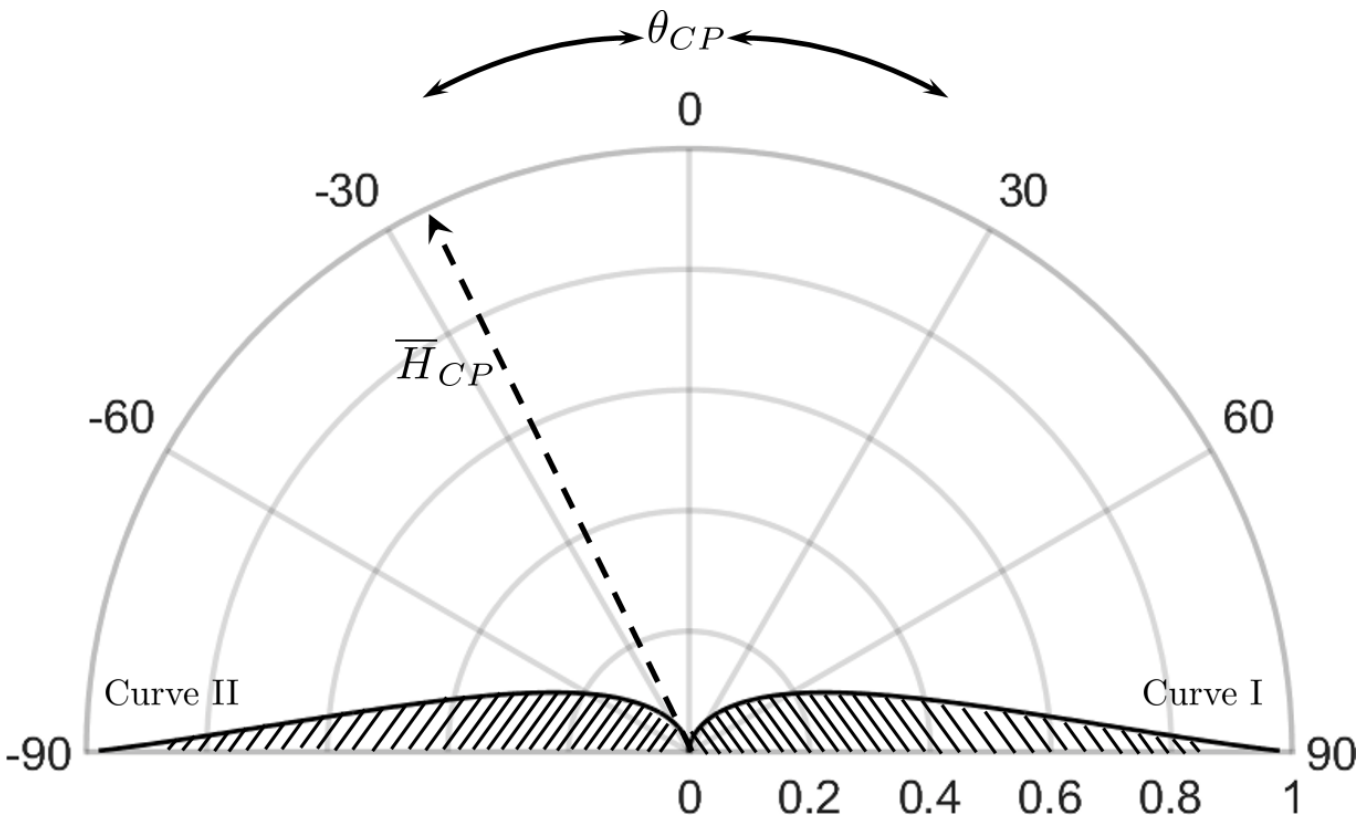

Figure 3: The $\bar{H}_{\mathrm{CP}} / \theta_{\mathrm{CP}}$ plane displayed in polar plot. Curve I and Curve II represent the azimuthal symmetry lines. No scattering mechanisms exist in the shaded portion of the plane. 
regions in the $\bar{H}_{\mathrm{CP}} / \theta_{\mathrm{CP}}$ polar plot is represented by two bounding curves, Curve I and Curve II in figure 3.

$$
\begin{gathered}
\text { Curve I, }[\mathbf{C}]_{I}=\left[\begin{array}{cc}
\frac{2 m+1}{4} & i \frac{2 m-1}{4} \\
-i \frac{2 m-1}{4} & \frac{2 m+1}{4}
\end{array}\right] \quad 0 \leq m \leq 0.5 \\
\text { Curve II, }[\mathbf{C}]_{I I}=\left[\begin{array}{cc}
\frac{2 m+1}{4} & -i \frac{2 m-1}{4} \\
i \frac{2 m-1}{4} & \frac{2 m+1}{4}
\end{array}\right] \quad 0 \leq m \leq 0.5
\end{gathered}
$$

\subsection{Clustering}

In this study, we propose clustering schemes equivalently for both $\mathrm{FP}$ and CP SAR data by utilizing the $2 \mathrm{D} \bar{H}_{\mathrm{FP}} / \theta_{\mathrm{FP}}$ and $\bar{H}_{\mathrm{CP}} / \theta_{\mathrm{CP}}$ planes respectively. Besides, the zones and the boundaries of both the clustering planes are identical. From analysis with scattering model (random volume model), it has been observed that the scattering-type from vegetation lies approximately in the range $-10^{\circ}$ to $20^{\circ}$ (Antropov et al., 2011). The upper bound for multiple scattering $\left(\theta_{X}=20^{\circ}\right.$, where the subscript $X$ refers to either FP or $\mathrm{CP}$ ) is characterized by equal contributions from the ensemble of horizontal and vertical dipole scattering components from vegetation structure. In contrast, the lower bound $\left(\theta_{X}=-10^{\circ}\right)$ is the characteristic of multiple scattering phenomena predominantly described by vertical vegetation structure. Hence, this region is subdivided for multiple scattering mechanisms. Unlike the $H / \bar{\alpha}$ plane, the proposed clustering scheme divides the plane into twelve zones. The scattering-type parameter $\theta_{X}$ divides the $\bar{H}_{X}-\theta_{X}$ plane into four sub-planes (P1:(Z1, Z2, Z3); P2:(Z4, Z5, Z6); P3:(Z7, Z8, Z9); P4:(Z10, Z11, Z12)) which consists of (1) pure even-bounce scattering $\left(-90^{\circ}\right.$ to $\left.-10^{\circ}\right)$ in $\mathrm{P} 1$; (2) even-bounce with multiple scattering $\left(-10^{\circ}\right.$ to $\left.0^{\circ}\right)$ in P2; (3) odd-bounce 


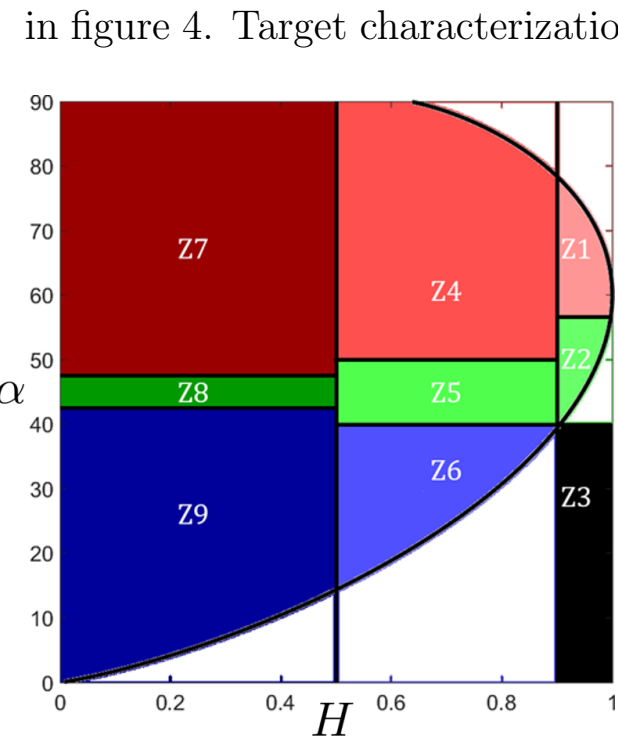

(a) $H / \bar{\alpha}$

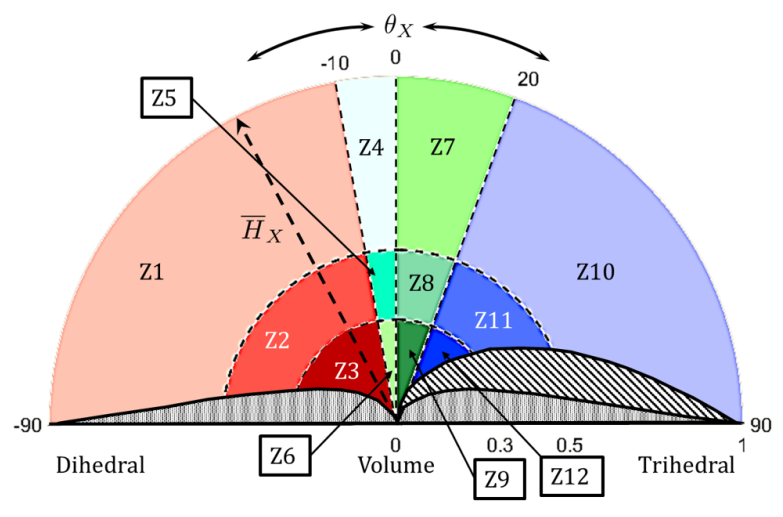

(b) $\bar{H}_{X} / \theta_{X}$

Figure 4: (a) $H / \bar{\alpha}$ clustering plane for FP SAR data with 8 clusters and (b) $\bar{H}_{X} / \theta_{X}$ clustering plane for both FP and CP SAR data with 12 clusters. Two half-circles at 0.3 and 0.5 divide $\bar{H}_{X}$ into high, medium and low entropy regions while $-90^{\circ}$ to $-10^{\circ}$ represents even bounce scattering, $-10^{\circ}$ to $20^{\circ}$ represents multiple bounce scattering and $20^{\circ}$ to $90^{\circ}$ represents odd bounce scattering. No scattering mechanisms exist in the shaded portion of the plane for both the FP and CP modes.

framework are implemented using MATLAB R2019b environment (the steps along with a flowchart are detailed in Appendix C). The proposed clustering framework is analyzed for the C-band San-Francisco RADARSAT-2 SAR data and further utilized for phenology clustering of rice. 


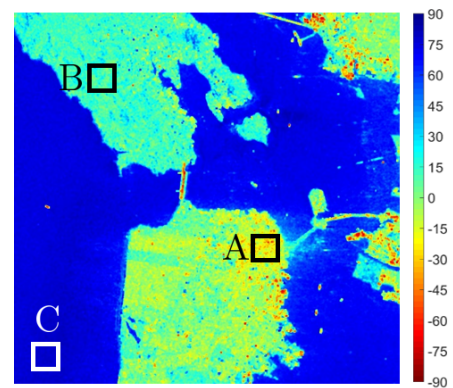

(a) $\bar{\alpha}$

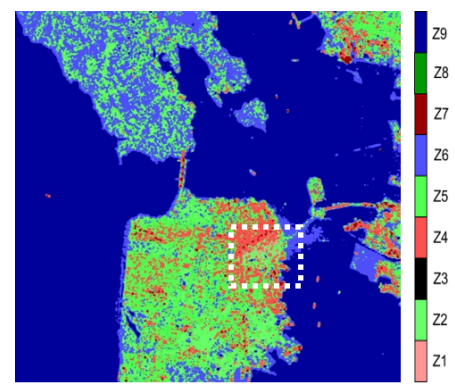

(d) $H / \bar{\alpha}$

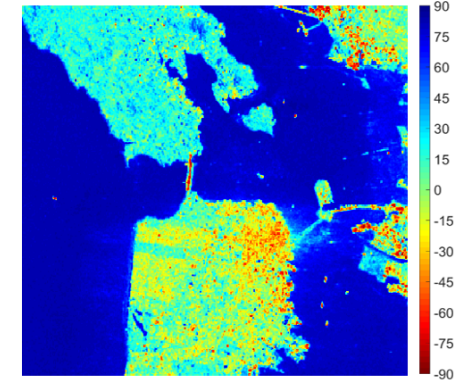

(b) $\theta_{\mathrm{FP}}$

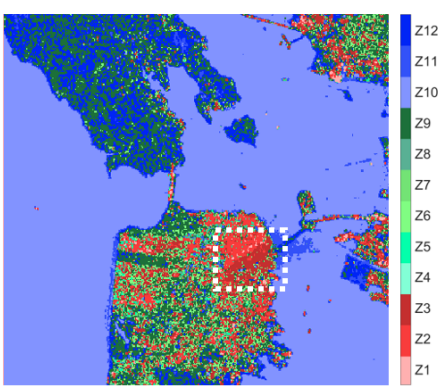

(e) $\bar{H}_{F P} / \theta_{F P}$

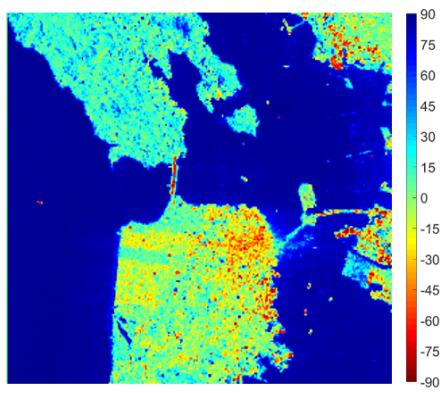

(c) $\theta_{\mathrm{CP}}$

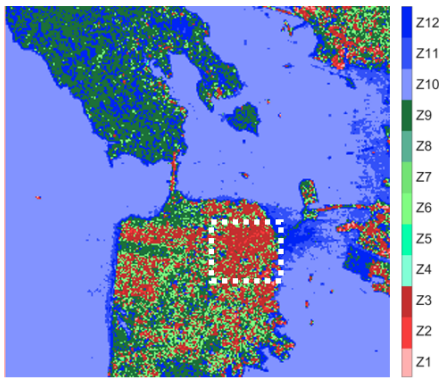

(e) $\bar{H}_{C P} / \theta_{C P}$

Figure 5: The scattering type parameters, $\bar{\alpha}, \theta_{\mathrm{FP}}, \theta_{\mathrm{CP}}$ and the $H / \bar{\alpha}, \bar{H}_{\mathrm{FP}} / \theta_{\mathrm{FP}}, \bar{H}_{\mathrm{CP}} / \theta_{\mathrm{CP}}$ clustered image of San Francisco Bay, USA using C-band RADARSAT-2 SAR data. Region $\mathrm{A}$ represents the oriented urban area, region $\mathrm{B}$ and $\mathrm{C}$ represents forest and ocean areas, respectively. The white box shows the oriented urban area where the major change during clustering occured. $H / \bar{\alpha}$ identified it as scattering from vegetation while $\bar{H}_{\mathrm{FP}} / \theta_{\mathrm{FP}}$ and $\bar{H}_{\mathrm{CP}} / \theta_{\mathrm{CP}}$ correctly identified it as scattering from urban region.

The difference between the geometrical structures of the $H / \bar{\alpha}$ and $\bar{H}_{\mathrm{FP}} / \theta_{\mathrm{FP}}$

$2 \mathrm{D}$ clustering planes can be observed in figure 4. As stated earlier, it may 


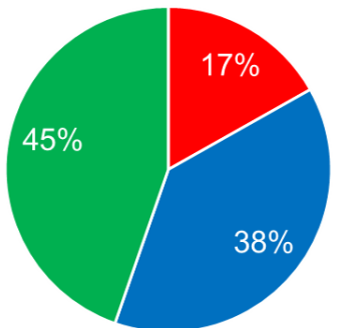

(a) $H / \bar{\alpha}$

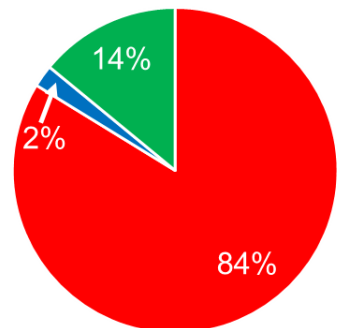

(b) $\bar{H}_{F P} / \theta_{F P}$

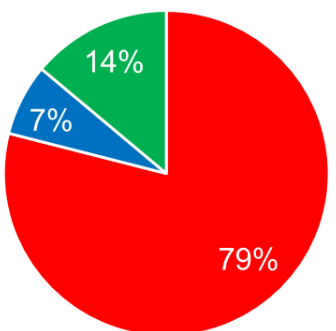

(b) $\bar{H}_{C P} / \theta_{C P}$

even bounce odd bounce

multiple bounce

(a) Rotated urban

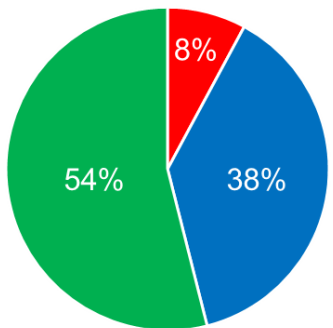

(a) $H / \bar{\alpha}$

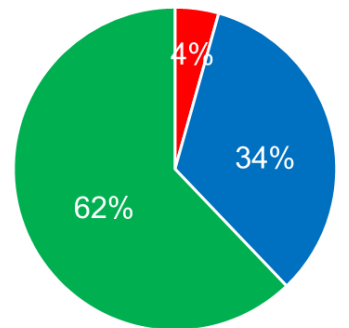

(b) $\bar{H}_{F P} / \theta_{F P}$

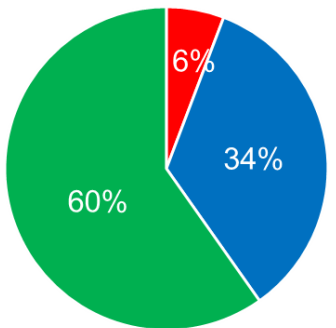

(b) $\bar{H}_{C P} / \theta_{C P}$

even bounce

odd bounce

multiple bounce

(b) Forest

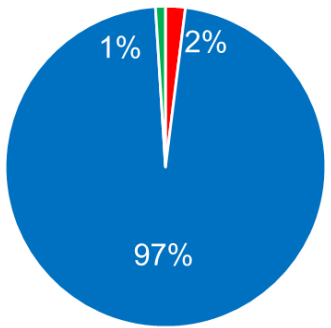

(a) $H / \bar{\alpha}$

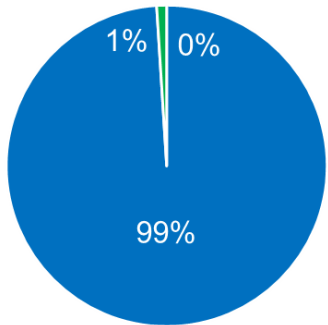

(b) $\bar{H}_{F P} / \theta_{F P}$

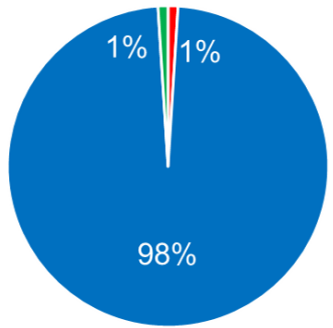

(b) $\bar{H}_{C P} / \theta_{C P}$

even bounce

multiple bounce

(c) Ocean

Figure 6: A comparison of the percentages of even, odd and multiple bounce scattering over (a) rotated urban, (b) forest and (c) ocean surfaces for the C-band RADARSAT-2 San Francisco Bay area image using $H / \bar{\alpha}, \bar{H}_{\mathrm{FP}} / \theta_{\mathrm{FP}}$ and $\bar{H}_{\mathrm{CP}} / \theta_{\mathrm{CP}}$ clustering techniques.

${ }_{334}$ be noted that the parameter $\bar{\alpha}$ is scaled to $\widehat{\alpha}=90^{\circ}-2 \bar{\alpha}$ solely for the sake of qualitative comparison. The ability of the two clustering planes, i.e., 
$\bar{H}_{\mathrm{FP}} / \theta_{\mathrm{FP}}$ and $\bar{H}_{\mathrm{CP}} / \theta_{\mathrm{CP}}$ to classify different land-cover classes is apparent in this figure. Region A, B and C in figure 5 are respectively the oriented urban area, forest area and ocean areas. The dashed white box in figure 5 highlights distinct changes in the scattering types as well as the clustering zones for differently oriented targets.

It can be observed from figure 6 that in the $H / \bar{\alpha}$ plane, the even-bounce scattering mechanism over oriented urban area (A) is only $17 \%$ while the odd-bounce and multiple-bounce scattering mechanism are $38 \%$ and $45 \%$, respectively. In contrast, the contribution of even-bounce dominant scattering mechanism in $\bar{H}_{\mathrm{FP}} / \theta_{\mathrm{FP}}$ and $\bar{H}_{\mathrm{CP}} / \theta_{\mathrm{CP}}$ are $84 \%$ and $79 \%$, respectively. On the other hand, over the forest area (B), the multiple-bounce scattering mechanism is $8 \%$ higher for $\bar{H}_{\mathrm{FP}} / \theta_{\mathrm{FP}}$ and $6 \%$ higher for $\bar{H}_{\mathrm{CP}} / \theta_{\mathrm{CP}}$ as compared to $H / \bar{\alpha}$. Similarly, over the ocean area $(\mathrm{C})$ the odd-bounce scattering mechanism has increased marginally by $2 \%$ and $1 \%$ for $\bar{H}_{\mathrm{FP}} / \theta_{\mathrm{FP}}$ and $\bar{H}_{\mathrm{CP}} / \theta_{\mathrm{CP}}$, respectively.

This suggests that the discriminating ability of $\bar{H}_{\mathrm{FP}} / \theta_{\mathrm{FP}}$ and $\bar{H}_{\mathrm{CP}} / \theta_{\mathrm{CP}}$ scheme is by and large higher than $H / \bar{\alpha}$. This marked ability might be due to 1) the joint utilization of the Barakat degree of polarization along with essential information from elements of the coherency matrix in deriving the scattering-type parameters, 2) the notion of an extended clustering procedure (i.e., 12 clusters) using entropy and the scattering-type parameters. Hence, we use the proposed clustering schemes with $\theta_{\mathrm{FP}}$ and $\theta_{\mathrm{CP}}$, for the temporal analysis of two different varieties of rice crops over Vijayawada, India using FP RADARSAT-2 data and simulated CP SAR data. In this study, we analyze the phenological changes of rice using these parameters and the new 
clustering scheme.

\subsection{Temporal variations of $\theta_{X}$ and $\bar{H}_{X} / \theta_{X}$ clustering planes for rice}

In this section, we discuss the temporal analysis of $\theta_{\mathrm{FP}}$ and $\theta_{\mathrm{CP}}$ along with phenology clustering of rice in the $\bar{H}_{\mathrm{FP}} / \theta_{\mathrm{FP}}$ and $\bar{H}_{\mathrm{CP}} / \theta_{\mathrm{CP}}$ polar plane. The temporal variation of $\theta_{\mathrm{FP}}$ and $\theta_{\mathrm{CP}}$ for FP and CP SAR data, respectively, are shown in figure 7. Both qualitative and quantitative analyses of the temporal variations in $\theta_{\mathrm{FP}}$ and $\theta_{\mathrm{CP}}$ utilizing data from five in-situ points (viz., P012, P054, P064, P034, and P053) are shown in figure 8. Besides, the $\bar{H}_{\mathrm{FP}} / \theta_{\mathrm{FP}}$ and $\bar{H}_{\mathrm{CP}} / \theta_{\mathrm{CP}}$ planes are divided into 12 zones based on different scattering-type information. In figure 4, zones (Z1, Z2, Z3), (Z10, Z11, Z12), and (Z4, Z5, Z6, Z7, Z8, Z9) represent even, odd and multiple scattering types respectively. In this study, these clustering zones (figure 16) are utilized to monitor the growth stages of rice using full and simulated compact polartimetric SAR data. The temporal variations of the clusters are shown in figures 9 to 14 . In figure $9 \mathrm{a}$ and figure $9 \mathrm{~b}$, the $\theta_{\mathrm{FP}}$ and $\theta_{\mathrm{CP}}$ values are majorly within the odd-bounce scattering region on 05 Jul depending on soil surface condition. Although the overall values of $\theta_{\mathrm{FP}}$ and $\theta_{\mathrm{CP}}$ are comparable, the FP image can better capture the subtle variations over the land cover compared to the CP image. During this period, the field condition differs depending on the ploughed and non-ploughed situation. The comparatively low values $\left(\leq 90^{\circ}\right)$ for $\theta_{\mathrm{FP}}$ and $\theta_{\mathrm{CP}}$ are likely due to soil roughness as compared to the incident 5.6 cm C-band wavelength (Mandal et al., 2020). Hence, dense clusters are seen in Z10, Z11, and Z12, which corresponds respectively to low entropy evenbounce scattering, medium entropy even-bounce scattering, and high entropy even-bounce scattering regions. Moreover, a few data points lying in region 


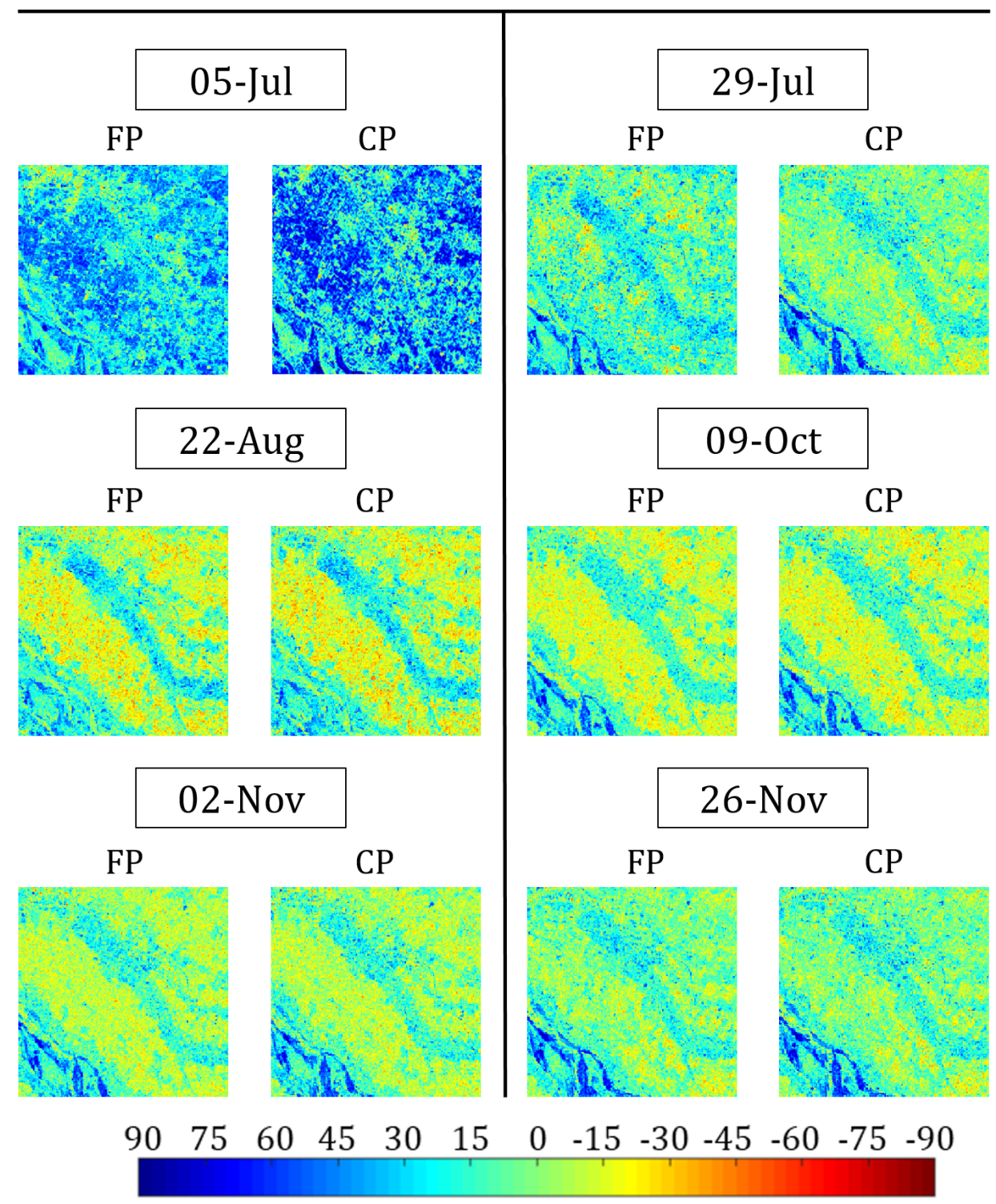

Figure 7: Variation of $\theta_{\mathrm{FP}}$ and $\theta_{\mathrm{CP}}$ images for $\mathrm{FP}$ and $\mathrm{CP}$ over the study area. The growth stages are: 5-Jul: Bare field, 29-Jul: Early tillering, 22-Aug: Advanced tillering, 9-Oct: Flowering, 2-Nov: Early dough, and 26-Nov: Maturity

$\mathrm{Z} 3$ is due to the early transplantation stage. Besides, tillage operation in some fields has produced soil surface roughness, which increased the entropy, 


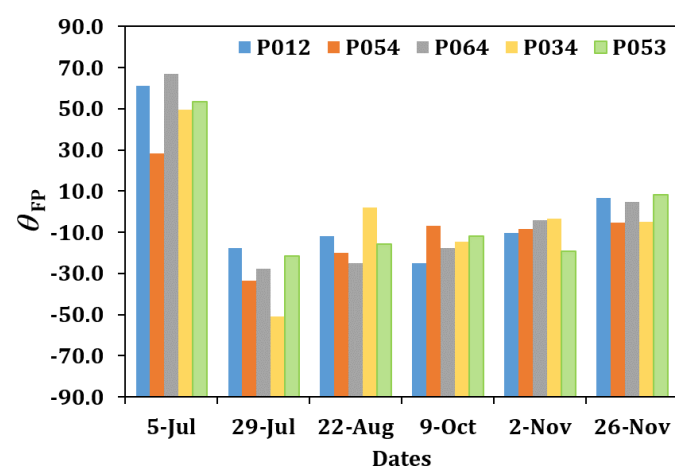

(a) FP data

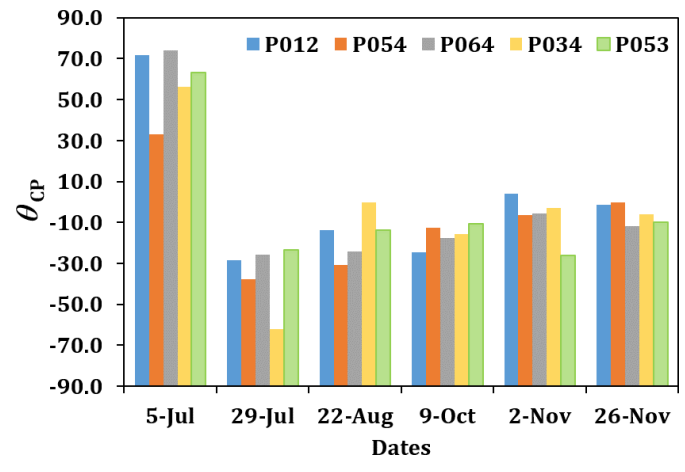

(b) CP data

Figure 8: Temporal variation of $\theta_{\mathrm{FP}}$ and $\theta_{\mathrm{CP}}$ for rice using $\mathrm{FP}$ and $\mathrm{CP}$ data for five in-situ points: P012, P054, P064, P034, and P053. The growth stages are: 5-Jul: Bare field, 29Jul: Early tillering, 22-Aug: Advanced tillering, 9-Oct: Flowering, 2-Nov: Early dough, and 26-Nov: Maturity

and hence, a sparse cluster can also be seen in Z9 and Z6. The proportion of pixels over different scattering regions at each phenological stage is shown in Table 3 and figure 16. High odd bounce scattering (86.26\%) was noted for FP data. Besides, due to the slight roughness a small component of multiple bounce scattering (12.24\%) is observed during this period, whereas even bounce scattering contribution was only $0.90 \%$.

A significant change in the data cluster is seen on $29 \mathrm{Jul}$ (figure 10a and figure 10b). During this period, most of the rice fields were in the early tillering stage, while other non-cultivated fields had moist soil with high roughness that is evident from in-situ data. During this period, variation of $\theta_{\mathrm{FP}}$ is $-17^{\circ}$ to $-51^{\circ}$ while $\theta_{\mathrm{CP}}$ ranges from $-23^{\circ}$ to $-62^{\circ}$ as seen in figure 8. Also, this highly rough soil surface during this period has generated a high degree of randomness in the received EM wave, which resulted in an increased entropy. Hence, a shift from low entropy zone (Z10) to high (Z12) and medium (Z11) entropy zones is evident on 29 Jul. Also, some data 


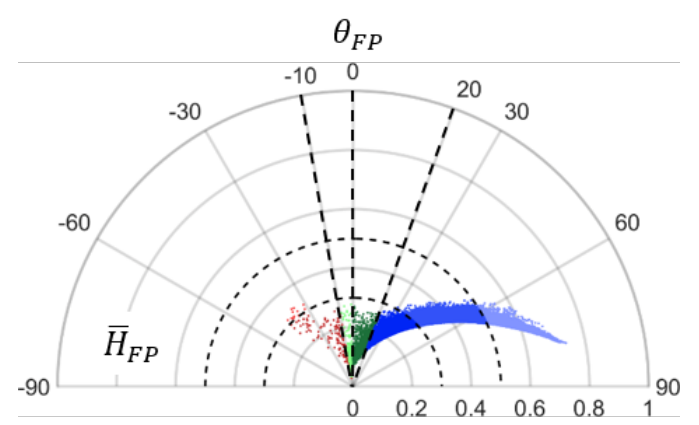

05-07-2018

(a) FP

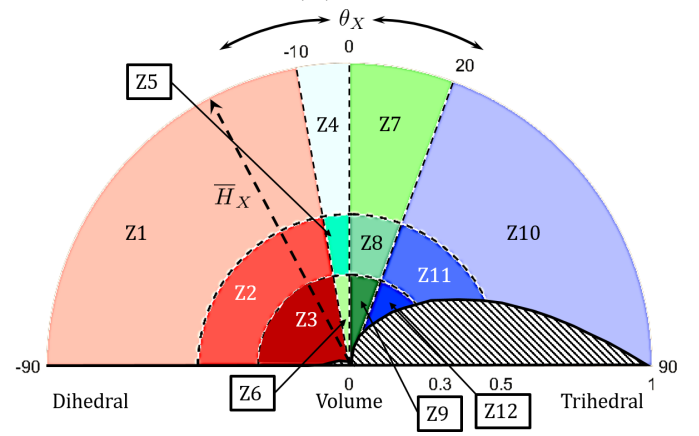

(c) Reference cluster (FP)

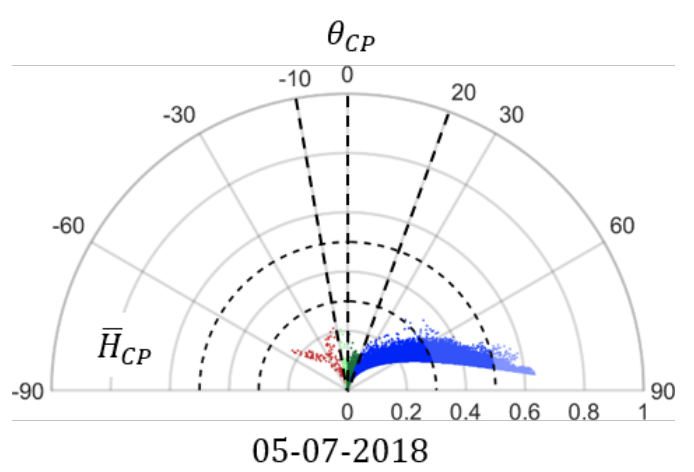

(b) $\mathrm{CP}$

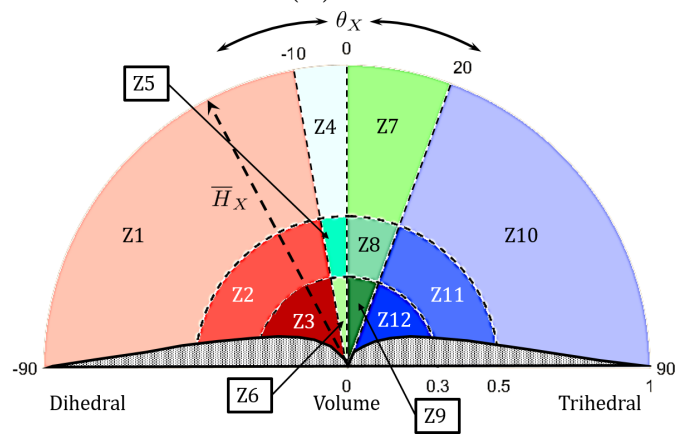

(d) Reference cluster (CP)

Figure 9: The $\bar{H}_{X} / \theta_{X}$ scatter plane for rice using FP and CP SAR data on 05-Jul.

points in zones Z11 and Z12 are $\theta_{\mathrm{FP}} \leq 30^{\circ}$, which is due to the scattering from the water surface in the rice fields (Lopez-Sanchez et al., 2014). However, compared to $\theta_{\mathrm{FP}}$, the values of $\theta_{\mathrm{CP}}$ are $5^{\circ}$ to $10^{\circ}$ higher in this period.

The density of the data points in Z6 and Z9 zones has also increased on $29 \mathrm{Jul}$, while rice transplantation was undergoing in some other fields. Therefore, a moderately high accumulation of data points can also be seen in Z3 (figure 10a and figure 10b). Moreover, the previously sown rice fields had achieved a higher vegetative stage due to which the areal coverage by the crop canopy had increased, thereby slightly decreasing the scattering entropy. 


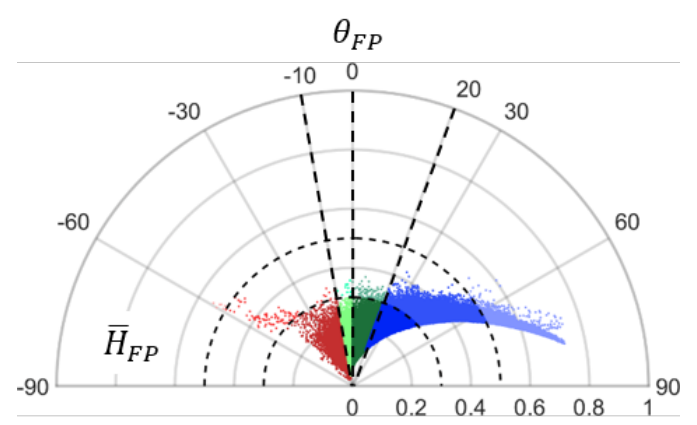

29-07-2018

(a) FP

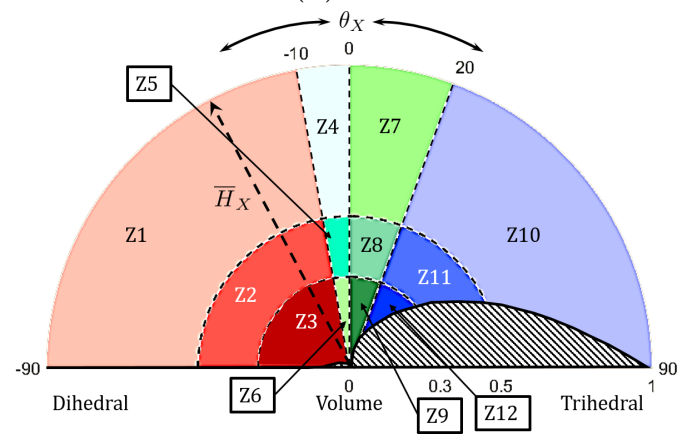

(c) Reference cluster (FP)

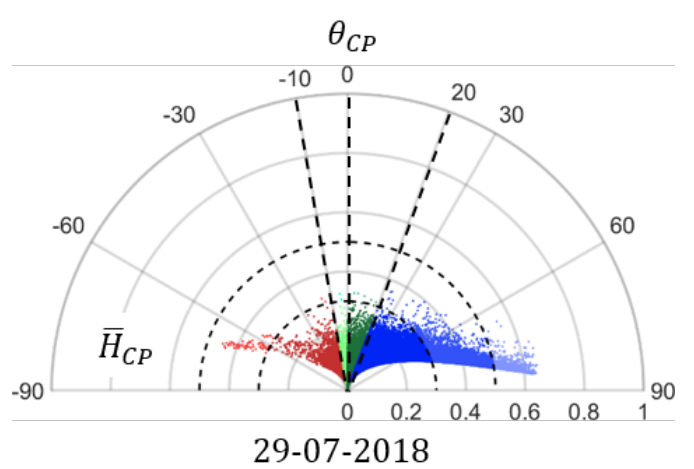

(b) $\mathrm{CP}$

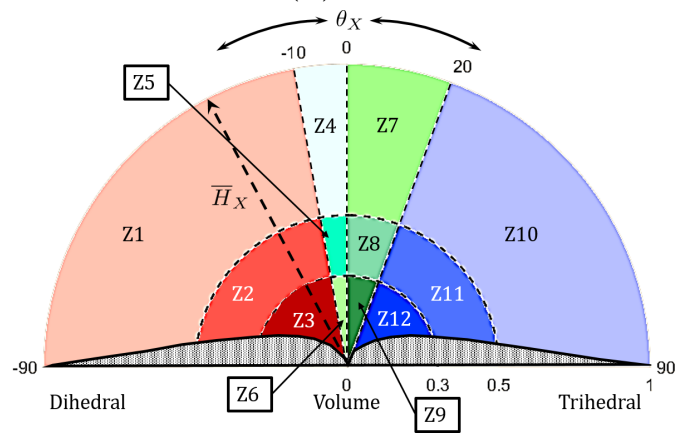

(d) Reference cluster (CP)

Figure 10: The $\bar{H}_{X} / \theta_{X}$ scatter plane for rice using FP and CP SAR data on 29-Jul.

Due to this aspect, a few data points are sparsely clustered in the Z2 region on $29 \mathrm{Jul}$. Furthermore, in zones $\mathrm{Z} 2$ and Z3, the values of $\theta_{\mathrm{CP}}$ is $2^{\circ}$ to $5^{\circ}$ higher than $\theta_{\mathrm{FP}}$. Hence, the even bounce scattering had increased by $75.89 \%$ and multiple scattering had increased by $16.49 \%$. A noteworthy decrease in the odd bounce scattering $(82.38 \%)$ is observed which is most likely due to the increase of double-bounce for the presence of stems, which also helps to reduce the surface roughness and the contribution from the ground.

On 22 Aug, dense clusters can be seen in Z3 for FP and CP data (figure $11 \mathrm{a}$ and figure $11 \mathrm{~b}$ ), which is due to the tillering stage of rice. Dur- 


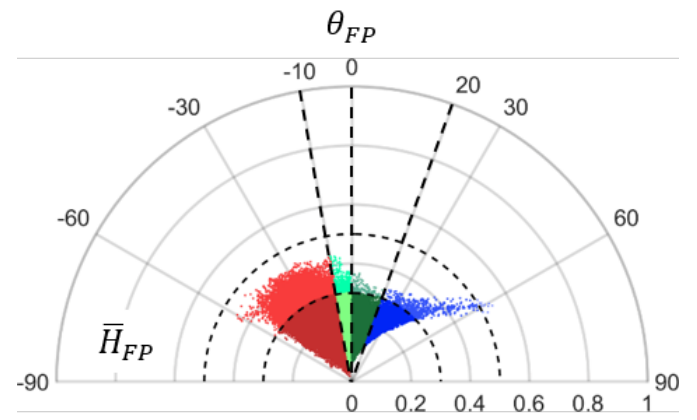

22-08-2018

(a) FP

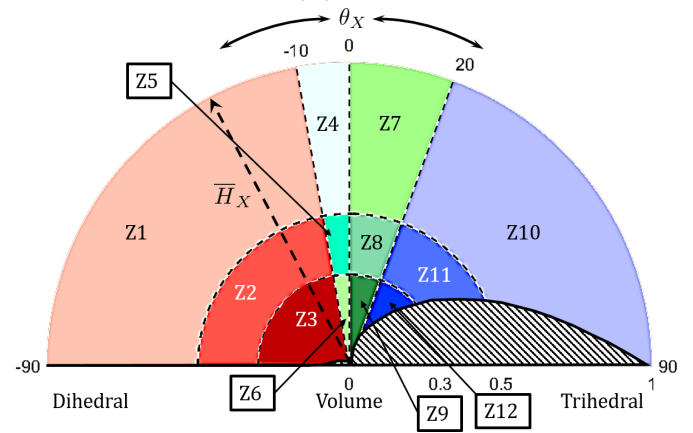

(c) Reference cluster (FP)

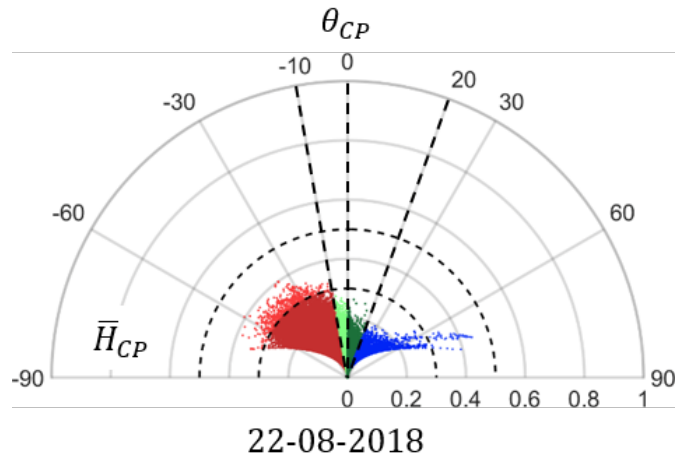

(b) $\mathrm{CP}$

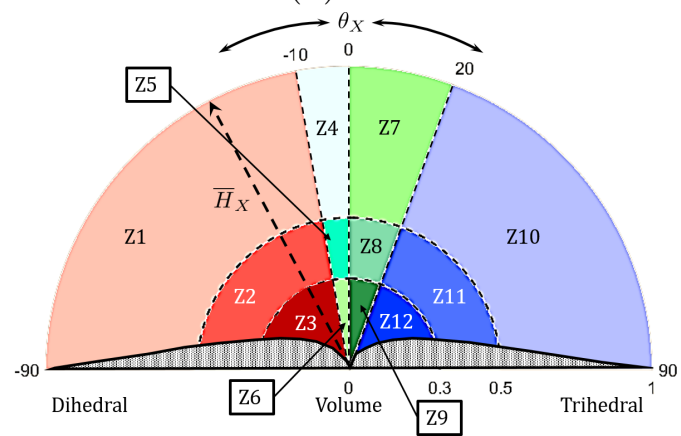

(d) Reference cluster (CP)

Figure 11: The $\bar{H}_{X} / \theta_{X}$ scatter plane for rice using FP and CP SAR data on 22-Aug.

ing this stage, the fields are flooded with water, and the stems are almost vertical, which acts as dihedral scatterers and generates even-bounce scattering (Yonezawa et al., 2012). Hence, a significant shift in the scattering mechanism from odd-bounce to even-bounce is visible during 22 Aug. During this period, $\bar{H}_{\mathrm{CP}}$ is lower than $\bar{H}_{\mathrm{FP}}$, which might be due to less polarimetric information content. Similar to $29 \mathrm{Jul}, \theta_{\mathrm{CP}}$ is higher than $\theta_{\mathrm{FP}}$ at this time. Additionally, due to the variation in the $\theta_{\mathrm{CP}}$ and $H_{\mathrm{CP}}$ values according to crop morphology, significant change among Z5, Z6, Z8, and Z9 zones can be observed compared to 29 Jul. Also, we observe an increasing trend in 
the plots in figure 8 due to the reduction in even-bounce multiple scattering. Besides, the orientation, shape, and size of each crop were not the same, and hence there was also a possibility of rough soil surface stretching out from the water surface. Therefore, these phenomena could induce high randomness in the scattered EM wave. Besides, similar to 29 Jul, some fields progressed to a higher vegetative stage due to which a cluster can be seen in Z2. Furthermore, fields that reached the booting stage display even-bounce multiple scattering due to which the even bounce scattering power had decreased by $11.19 \%$, while multiple bounce scattering had marginally increased by $3.67 \%$.

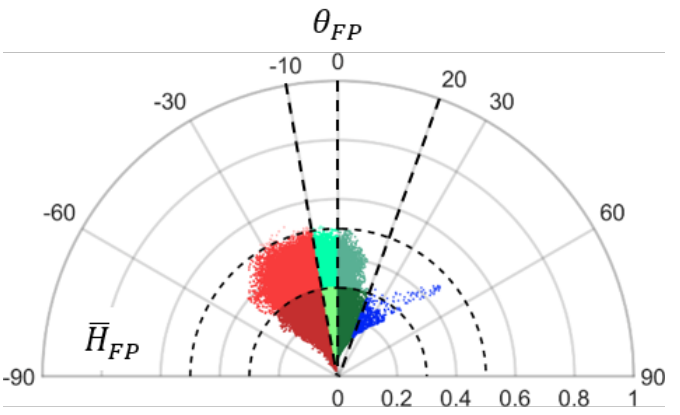

09-10-2018

(a) FP

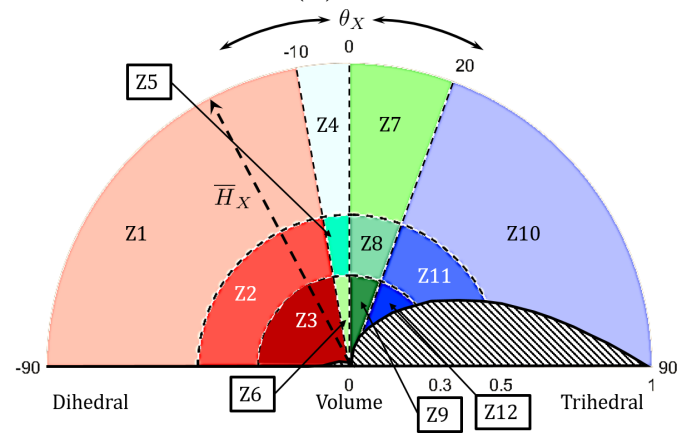

(c) Reference cluster (FP)

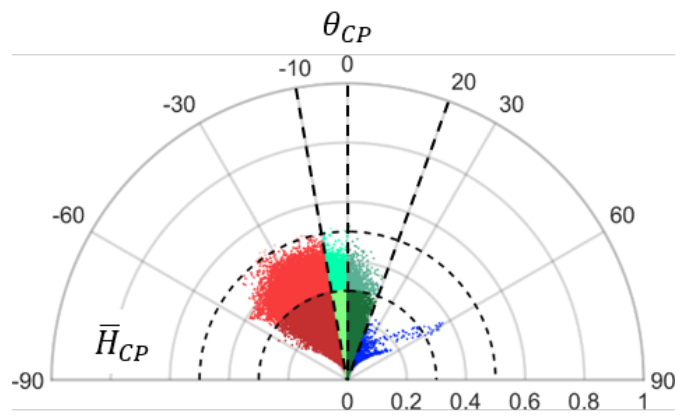

$09-10-2018$

(b) $\mathrm{CP}$

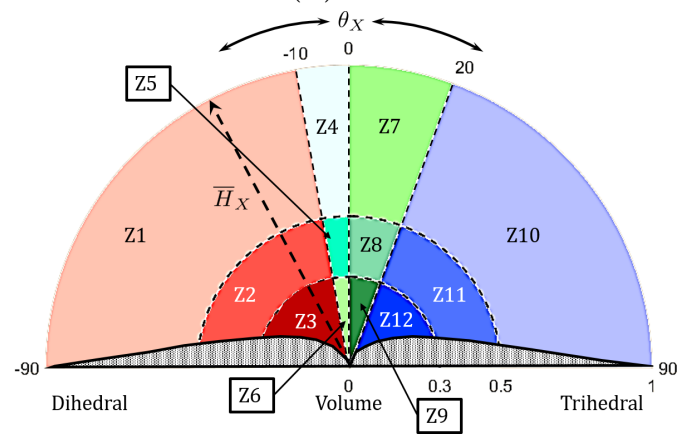

(d) Reference cluster (CP)

Figure 12: The $\bar{H}_{X} / \theta_{X}$ scatter plane for rice using FP and CP SAR data on 09-Oct. 
On 09 Oct, both $\bar{H}_{\mathrm{FP}} / \theta_{\mathrm{FP}}$ and $\bar{H}_{\mathrm{CP}} / \theta_{\mathrm{CP}}$ planes show a shift towards the medium entropy region (i.e., Z2 and Z5 zones) which is evident in figure $12 \mathrm{a}$ and figure 12b. During this period, most of the rice fields were in the inflorescence emergence stage, with $\theta_{\mathrm{FP}}$ and $\theta_{\mathrm{CP}}$ indicating even-bounce and even-bounce multiple scatterings. Moreover, the amount of cross-pol components has increased during this period and the coherence between the co-polarized channels decreased significantly. A similar type of increase in cross-pol components from transplantation to maturity stages was reported by He et al. (2018). The shift towards the Z2 and Z5 zones indicates an even-bounce scattering mechanism of the scattered EM wave. Such a response might be due to the extinction of the vertical polarization due to the canopy structure. Also, the amount of odd-bounce scattering reduced during this period, and rice foliage generated moderate odd-bounce multiple scattering due to which dense cluster in the Z8 zone is noticed. The contribution of multiple bounce scattering was $40.02 \%$ due to the full-grown rice crop with differently oriented stem, leaf structures and flowers.

Around 02 Nov, the rice fields reached the early dough stage, during which, the milky white substance begins to accumulate in rice panicle. Simultaneously, the crop water content during this period remains very high, while leaf and stem produce overall complex canopy structure, which leads to high randomness in the SAR backscatter. Due to this fact, the values of $\bar{H}_{\mathrm{FP}}$ and $\bar{H}_{\mathrm{CP}}$ are low. Moreover, at this point, the clusters in Z3 and Z2 zones are due to the scattering from compound leaf and stem structure (figure 13a and figure 13b). In contrast, clusters in Z6, Z5, Z8, and Z9 zones are due to multiple scattering contribution from the intermediate complex 


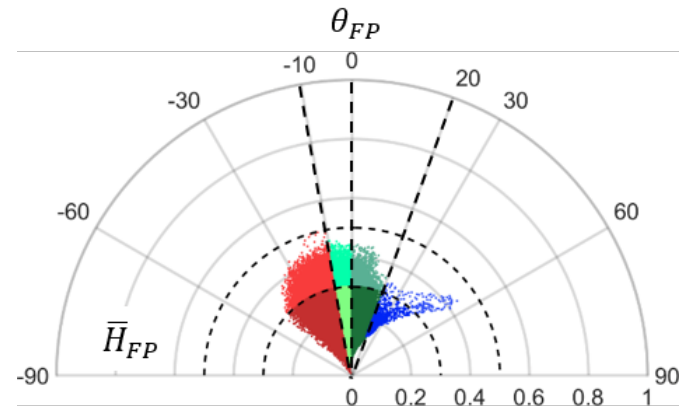

02-11-2018

(a) FP

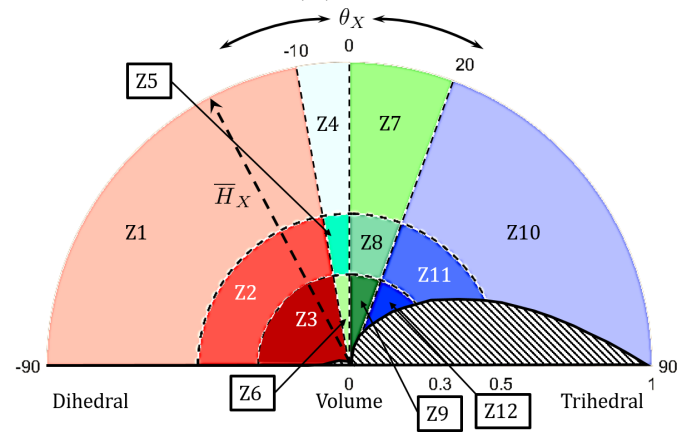

(c) Reference cluster (FP)

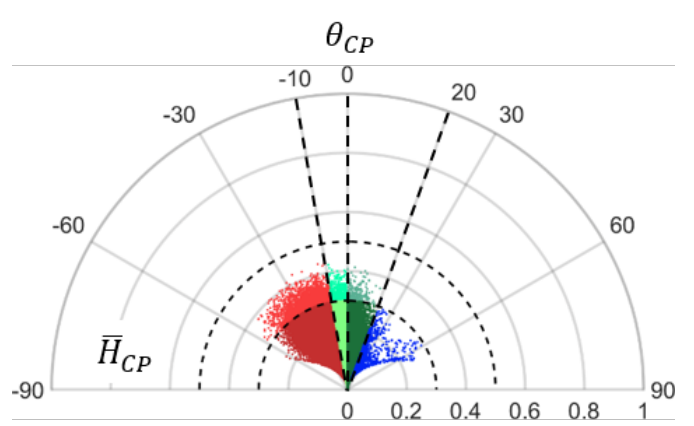

02-11-2018

(b) $\mathrm{CP}$

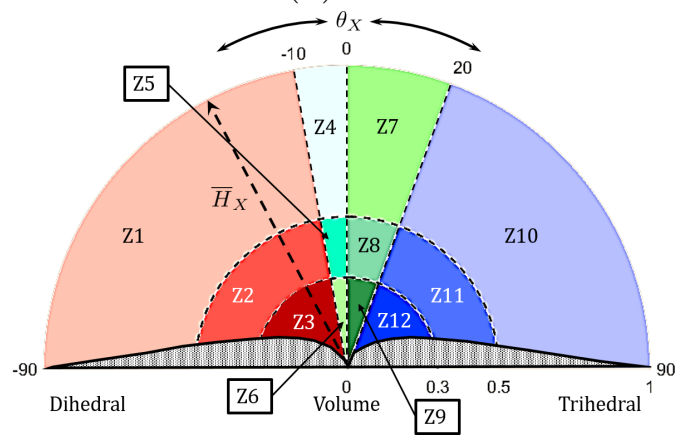

(d) Reference cluster (CP)

Figure 13: The $\bar{H}_{X} / \theta_{X}$ scatter plane for rice using FP and CP SAR data on 02-Nov.

rice canopy layer. The cluster in the $\mathrm{Z} 12$ zone corresponds to the scattering of the wave directly from the leaves of the uppermost canopy layer. During this time further decrease in even bounce scattering is evident.

On 26 Nov, the rice fields reached the maturity stage, and the grains become firm and heavy. At this point, the crop becomes dry, whereas the moisture content in grains remains $\approx 20 \%$. Due to the weight of the grains, lodging of rice is usually visible in the fields due to which the morphological condition becomes further complicated than the dough stage. Hence, an additional increase in the scattering entropy during this period is apparent for 


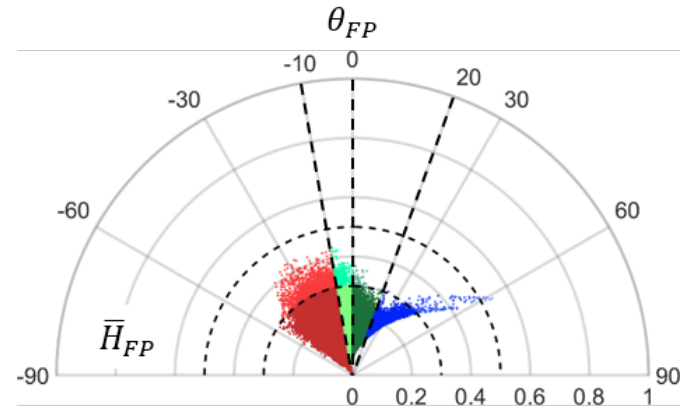

26-11-2018

(a) FP

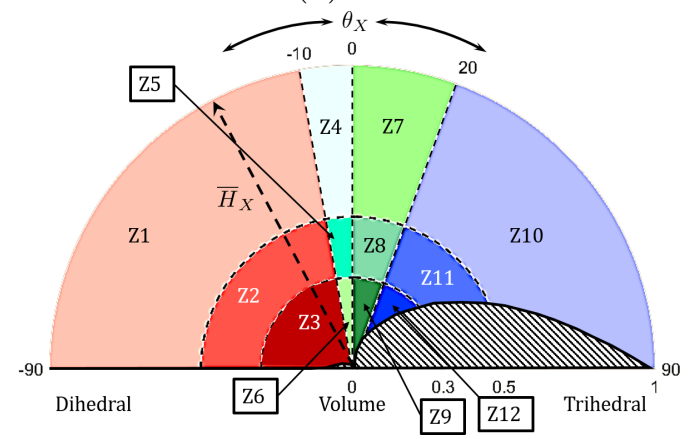

(c) Reference cluster (FP)

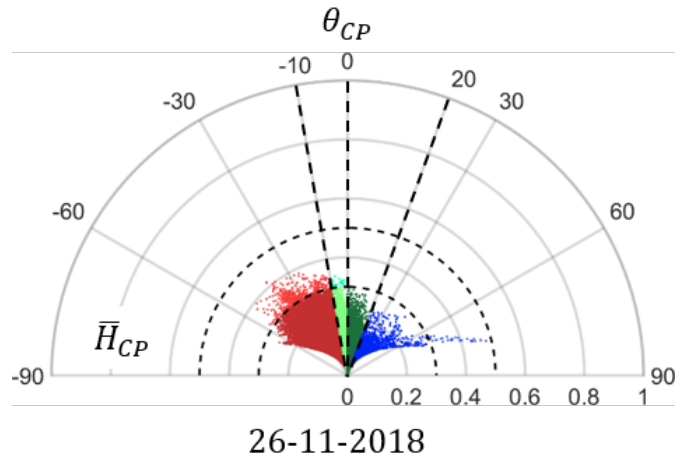

(b) $\mathrm{CP}$

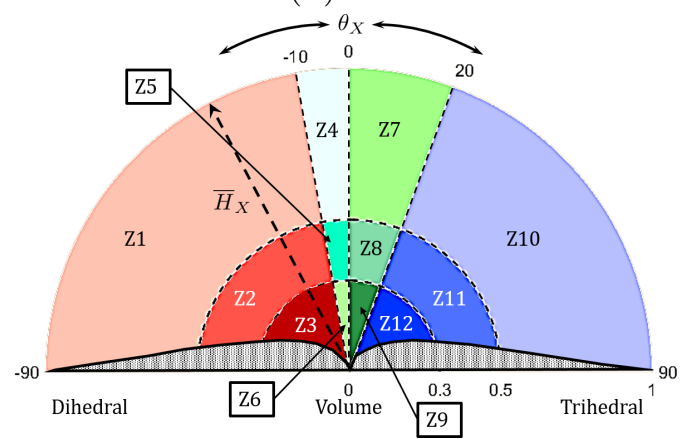

(d) Reference cluster (CP)

Figure 14: The $\bar{H}_{X} / \theta_{X}$ scatter plane for rice using FP and CP SAR data on 26-Nov.

both FP and CP SAR data. High densities of clusters in Z3, Z6, Z9, and Z12 zones can be noticed in figure 14a and figure 14b, which is due to scattering from the complex geometrical structure of rice at this stage. However, a small cluster can also be observed in the Z11 zone, which might be due to fully or partially harvested rice fields. At this stage, the highest contribution of multiple scattering mechanisms $(73.23 \%)$ is profound due to the increase in scattering randomness within the SAR resolution cell. We performed hypothesis testing to show that these changes in the scattering mechanisms for different dates are related to rice phenological changes. In this regard, the 
null hypothesis states that there exists no relationship between the changes in the clusters and rice phenology (i.e., the change is due to randomness). The $p$-values (95\% confidence level) as shown in Table 3 indicates that we can reject the null hypothesis, and therefore, there is evidence that the changes in the unsupervised clusters are due to rice phenology.

Table 3: Changes in the scattering mechanisms across different dates and between FP and $\mathrm{CP}$ data. we have considered (Z1, Z2, Z3) as even bounce scattering, (Z10, Z11, Z12) as odd bounce scattering and (Z4, Z5, Z6, Z7, Z8, Z9) as multiple bounce scattering. The dominant scattering mechanism(s) at each date is highlighted in bold font. Also, the $p$-values at $95 \%$ significance level is provided

\begin{tabular}{|c|c|c|c|c|c|c|}
\hline Dates & Modes & $\begin{array}{l}\text { Even bounce } \\
\text { scattering }\end{array}$ & $\begin{array}{c}\text { Odd bounce } \\
\text { scattering }\end{array}$ & $\begin{array}{l}\text { Multiple bounce } \\
\text { scattering }\end{array}$ & $\begin{array}{l}\text { Growth } \\
\text { Stage }\end{array}$ & $p$-value \\
\hline \multirow{2}{*}{$05 / 07 / 2018$} & $\mathrm{FP}$ & $0.90 \%$ & $86.86 \%$ & $12.24 \%$ & \multirow{2}{*}{ Bare field } & $2.30 \times 10^{-11}$ \\
\hline & $\mathrm{CP}$ & $0.60 \%$ & $88.28 \%$ & $11.12 \%$ & & $2.18 \times 10^{-11}$ \\
\hline \multirow{2}{*}{$29 / 07 / 2018$} & $\mathrm{FP}$ & $76.79 \%$ & $4.48 \%$ & $28.73 \%$ & \multirow{2}{*}{ Early tillering } & $2.20 \times 10^{-16}$ \\
\hline & $\mathrm{CP}$ & $64.60 \%$ & $2.10 \%$ & $33.30 \%$ & & $2.18 \times 10^{-15}$ \\
\hline \multirow{2}{*}{$22 / 08 / 2018$} & $\mathrm{FP}$ & $65.60 \%$ & $2 \%$ & $32.40 \%$ & \multirow{2}{*}{$\begin{array}{l}\text { Advanced } \\
\text { tillering }\end{array}$} & $2.20 \times 10^{-16}$ \\
\hline & $\mathrm{CP}$ & $63.87 \%$ & $2 \%$ & $34.13 \%$ & & $1.96 \times 10^{-16}$ \\
\hline \multirow{2}{*}{$09 / 10 / 2018$} & $\mathrm{FP}$ & $58.10 \%$ & $1.88 \%$ & $40.02 \%$ & \multirow{2}{*}{ Flowering } & $2.10 \times 10^{-16}$ \\
\hline & $\mathrm{CP}$ & $56.33 \%$ & $1.88 \%$ & $41.79 \%$ & & $2.10 \times 10^{-16}$ \\
\hline \multirow{2}{*}{$02 / 11 / 2018$} & $\mathrm{FP}$ & $39.40 \%$ & $3 \%$ & $57.60 \%$ & \multirow{2}{*}{ Early dough } & $2.40 \times 10^{-14}$ \\
\hline & $\mathrm{CP}$ & $31.60 \%$ & $2 \%$ & $66.40 \%$ & & $1.82 \times 10^{-14}$ \\
\hline \multirow{2}{*}{$26 / 11 / 2018$} & $\mathrm{FP}$ & $25.61 \%$ & $1.16 \%$ & $73.23 \%$ & \multirow{2}{*}{ Maturity } & $2.20 \times 10^{-16}$ \\
\hline & $\mathrm{CP}$ & $16.76 \%$ & $0.92 \%$ & & & $1.98 \times 10^{-16}$ \\
\hline
\end{tabular}

It is noteworthy that the differences in the characterization capability between FP and CP SAR data depends on the type and geometry of the targets. Moreover, the spatial heterogeneity induces the changes in the intensity of the co-pol and cross-pol components. Hence, a change in the scattered EM wave is sometimes evident between FP and CP SAR data.

\section{Conclusions}

In this study, we have proposed two scattering-type parameters, $\theta_{\mathrm{FP}}$ and $\theta_{\mathrm{CP}}$ for identifying target scattering mechanism for both full (FP) and com- 


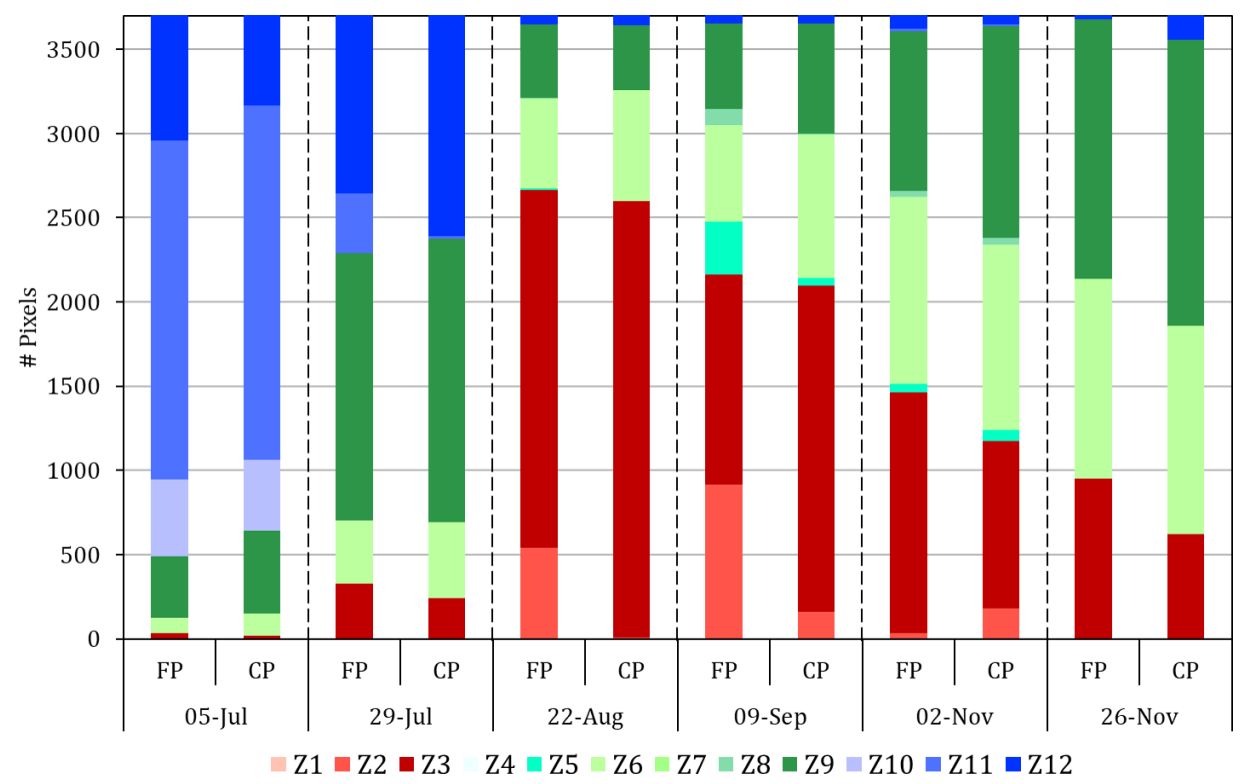

Figure 15: Variations in the number of pixels in different clusters for each date in FP and CP data.

pact polarimetric (CP) SAR data. These quantities are roll-invariant and vary in the range, $-90^{\circ}$ to $90^{\circ}$. In particular these two scattering-type parameters jointly utilize the received antenna basis-invariant parameters, i.e., the Barakat degree of polarization and the total scattering power (Span) and the elements of the coherency matrix. The two extreme values of their range correspond to even-bounce $\left(-90^{\circ}\right)$, and odd-bounce $\left(90^{\circ}\right)$ scattering mechanisms, while $\theta_{\mathrm{FP}}=0^{\circ}$ and $\theta_{\mathrm{CP}}=0^{\circ}$ denotes diffused scattering mechanism. Furthermore, $\theta_{\mathrm{FP}}$ and $\theta_{\mathrm{CP}}$ within the range, $-10^{\circ}$ to $0^{\circ}$ indicates even-bounce multiple scattering components, and $0^{\circ}$ to $20^{\circ}$ denotes the odd-bounce multiple scattering components.

In this study, we have suitably fulfilled our primary objective to characterize changes in the scattering mechanism with the advancement of crop phenological stages. We have used the scattering-type parameters for the 


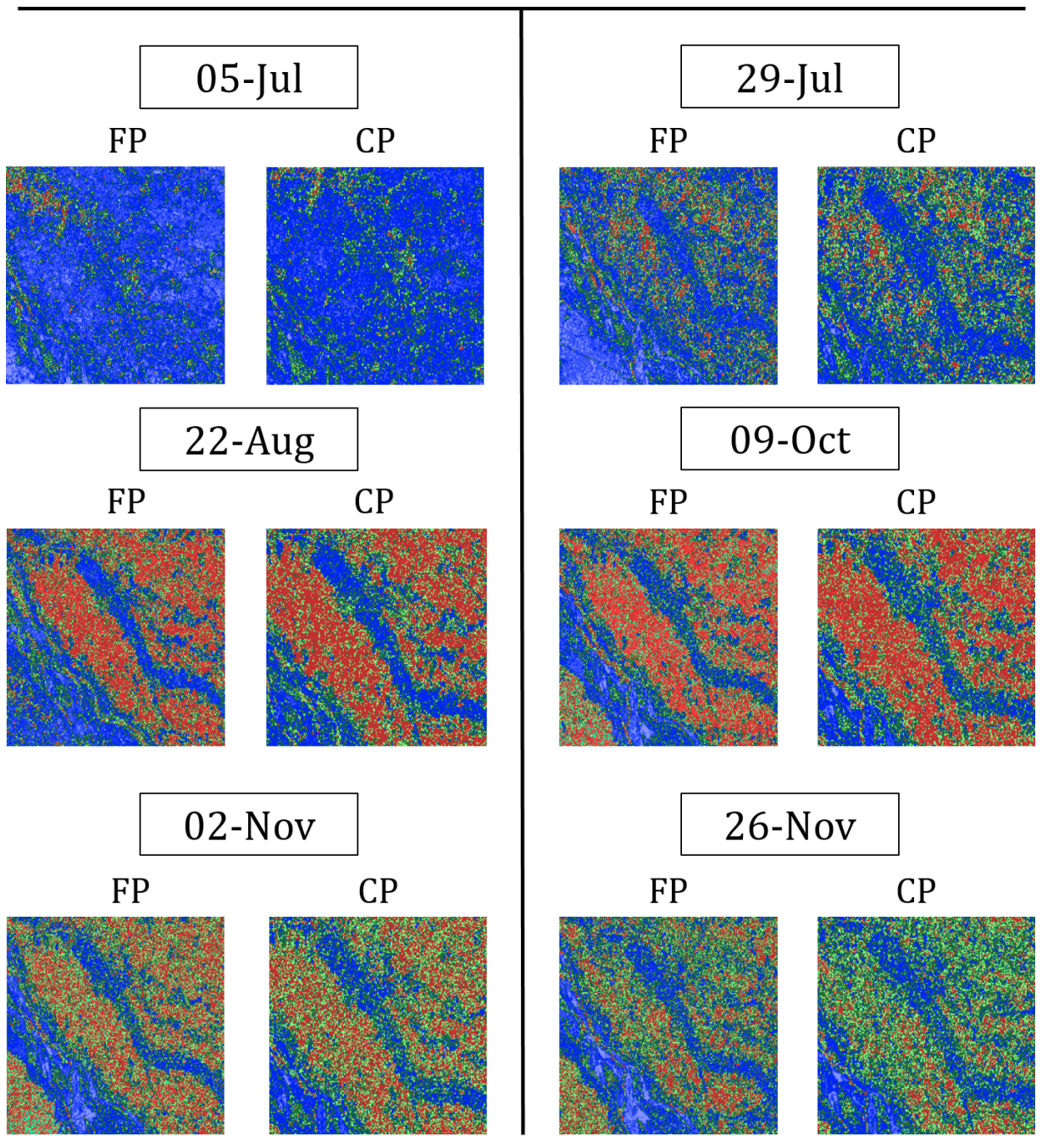

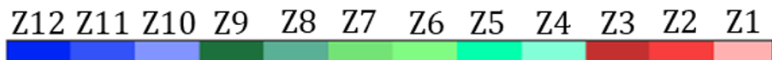

Figure 16: Variation of $\bar{H}_{\mathrm{FP}} / \theta_{\mathrm{FP}}$ and $\bar{H}_{\mathrm{CP}} / \theta_{\mathrm{CP}}$ clustered images for $\mathrm{FP}$ and $\mathrm{CP}$ over the study area. The growth stages are: 5-Jul: Bare field, 29-Jul: Early tillering, 22-Aug: Advanced tillering, 9-Oct: Flowering, 2-Nov: Early dough, and 26-Nov: Maturity

temporal analysis of rice over the Vijayawada test site in India using FP and CP SAR data. The sensitivities of $\theta_{\mathrm{FP}}$ and $\theta_{\mathrm{CP}}$ with growth stages of rice are significantly evident from this study. We have introduced novel new clus- 
tering schemes, $\bar{H}_{\mathrm{FP}} / \theta_{\mathrm{FP}}$ and $\bar{H}_{\mathrm{CP}} / \theta_{\mathrm{CP}}$ in this study by utilizing $\theta_{\mathrm{FP}}, \theta_{\mathrm{CP}}$, and the scattering entropies, $H_{\mathrm{FP}}$ and $H_{\mathrm{CP}}$. The clustering plane is split into 12 zones, where each zone represents a distinct dominant scattering mechanism. In this regard, the $\bar{H}_{\mathrm{FP}} / \theta_{\mathrm{FP}}$ and $\bar{H}_{\mathrm{CP}} / \theta_{\mathrm{CP}}$ clustering planes provide necessary information about targets without any apriori knowledge of the scene.

The target characterization parameters as well as the clustering planes provide information about changes in the scattering mechanism at different crop phenological stage. They could be beneficial in providing essential information about crop conditions for engaging different cultivation measures. Therefore, further investigation to track and map crop growth stages could be conducted for different crop-types around the globe. The sensitivity of these parameters for different crop geometry could be examined for different incident angles using both FP and CP SAR data. We could adequately utilize these parameters for the newly launched RADARSAT Constellation Mission (RCM) and several upcoming missions.

\section{Appendix A. Roll-invariant parameters}

A parameter which is independent of target orientation angle along the radar line of sight is called roll-invariant. In this section, we show the rollinvariant nature of $\theta_{\mathrm{FP}}$ and $\theta_{\mathrm{CP}}$. 
Appendix A.1. Roll-invariant nature of $\theta_{\mathrm{FP}}$

To show that $\theta_{\mathrm{FP}}$ is a roll-invariant parameter, let the coherency matrix $\mathbf{T}$ be unitarily rotated by $\mathbf{R}(\Psi)$ as,

$$
\mathbf{T}(\Psi)=\mathbf{R}(\Psi) \mathbf{T} \mathbf{R}(\Psi)^{-1}
$$

where

$$
\mathbf{R}(\Psi)=\left[\begin{array}{ccc}
1 & 0 & 0 \\
0 & \cos 2 \Psi & \sin 2 \Psi \\
0 & -\sin 2 \Psi & \cos 2 \Psi
\end{array}\right]
$$

with,

$$
\begin{aligned}
T_{11}(\Psi)= & T_{11} \\
T_{22}(\Psi)= & T_{22} \cos ^{2}(2 \Psi)+T_{32} \cos (2 \Psi) \sin (2 \Psi)+ \\
& T_{23} \cos (2 \Psi) \sin (2 \Psi)+T_{33} \sin ^{2}(2 \Psi) \\
T_{33}(\Psi)= & T_{22} \sin ^{2}(2 \Psi)-T_{32} \cos (2 \Psi) \sin (2 \Psi)- \\
& T_{23} \cos (2 \Psi) \sin (2 \Psi)+T_{33} \cos ^{2}(2 \Psi)
\end{aligned}
$$

Therefore, $T_{11}(\Psi)-T_{22}(\Psi)-T_{33}(\Psi)=T_{11}-T_{22}-T_{33}$ and $T_{22}(\Psi)+T_{33}(\Psi)=$ $T_{22}+T_{33}$ i.e., both $T_{11}-T_{22}-T_{33}$ and $T_{22}+T_{33}$ are independent of the unitary rotation by an angle $\Psi$. Alongside this, note that the total power i.e., Span $=T_{11}(\Psi)+T_{22}(\Psi)+T_{33}(\Psi)=T_{11}+T_{22}+T_{33}=\operatorname{Tr}(\mathbf{T})$, and $|\mathbf{T}|$ are roll-invariant, where $|\cdot|$ is the determinant and $\operatorname{Tr}(\cdot)$ is the trace of a matrix.

Therefore, the 3D Barakat degree of polarization, $m_{\mathrm{FP}}=\sqrt{1-\frac{27|\mathbf{T}|}{(\operatorname{Tr}(\mathbf{T}))^{3}}}$ is also independent of $\Psi$. Hence, we conclude that the proposed scattering-type 
543 parameter for FP SAR,

$$
\theta_{\mathrm{FP}}=2 \tan ^{-1}\left(\frac{m_{\mathrm{FP}} \operatorname{Span}\left(T_{11}-T_{22}-T_{33}\right)}{T_{11}\left(T_{22}+T_{33}\right)+m_{\mathrm{FP}}^{2} \operatorname{Span}^{2}}\right)
$$

544 is independent of $\Psi$, i.e., it is a roll-invariant parameter.

${ }_{545}$ Appendix A.2. Roll-invariant nature of $\theta_{\mathrm{CP}}$

${ }_{546}$ The $2 \times 2$ covariance matrix can be expressed in terms of the elements of ${ }_{547}$ the Stokes vector $\overrightarrow{\mathbf{S}}=\left[S_{0}, S_{1}, S_{2}, S_{3}\right]$ as

$$
\mathbf{C}_{2}=\frac{1}{2}\left[\begin{array}{cc}
S_{0}+S_{1} & S_{2}+i S_{3} \\
S_{2}-i S_{3} & S_{0}-S_{1}
\end{array}\right]
$$

${ }_{548}$ Let the $\mathbf{C}_{2}$ matrix be unitarily rotated by $\mathbf{R}(\Psi)$ as $\mathbf{C}_{2}(\Psi)=\mathbf{R}(\Psi) \mathbf{C}_{2} \mathbf{R}(\Psi)^{-1}$, 549 where the rotation matrix is,

$$
\mathbf{R}(\Psi)=\left[\begin{array}{cc}
\cos (\Psi) & -\sin (\Psi) \\
\sin (\Psi) & \cos (\Psi)
\end{array}\right]
$$


The elements of the $\mathbf{C}_{2}(\Psi)$ matrix are:

$$
\begin{aligned}
c_{11}(\Psi)= & \cos ^{2} \Psi\left(S_{0}+S_{1}\right)-\cos \Psi \sin \Psi\left(S_{2}-i S_{3}\right)- \\
& \cos \Psi \sin \Psi\left(S_{2}+i S_{3}\right)+\sin ^{2} \Psi\left(S_{0}-S_{1}\right) \\
c_{12}(\Psi)= & \cos \Psi \sin \Psi\left(S_{0}+S_{1}\right)-\sin ^{2} \Psi\left(S_{2}-i S_{3}\right)+ \\
& \cos ^{2} \Psi\left(S_{2}+i S_{3}\right)-\cos \Psi \sin \Psi\left(S_{0}-S_{1}\right) \\
c_{21}(\Psi)= & \cos \Psi \sin \Psi\left(S_{0}+S_{1}\right)+\cos ^{2} \Psi\left(S_{2}-i S_{3}\right)- \\
& \sin ^{2} \Psi\left(S_{2}+i S_{3}\right)-\cos \Psi \sin \Psi\left(S_{0}-S_{1}\right) \\
c_{22}(\Psi)= & \sin ^{2} \Psi\left(S_{0}+S_{1}\right)-\cos \Psi \sin \Psi\left(S_{2}-i S_{3}\right)- \\
& \cos \Psi \sin \Psi\left(S_{2}+i S_{3}\right)+\cos ^{2} \Psi\left(S_{0}-S_{1}\right) .
\end{aligned}
$$

The total power $S_{0}=c_{11}(\Psi)+c_{22}(\Psi)$ and the fourth element of the Stokes vector $S_{3}=-i\left(c_{12}(\Psi)-c_{21}(\Psi)\right)$ are independent of the rotation angle $\Psi$. Since $S_{0}$ and $S_{3}$ are independent of $\Psi$, then $\mathrm{SC}=\left(S_{0}-S_{3}\right) / 2$ and $\mathrm{OC}=$ $\left(S_{0}+S_{3}\right) / 2$ are also independent of $\Psi$, i.e. both parameters are roll-invariant. Alongside this, note that $\left|\mathbf{C}_{2}\right|$ and $\operatorname{Tr}\left(\mathbf{C}_{2}\right)$ are roll-invariant, where $|\cdot|$ is the determinant and $\operatorname{Tr}(\cdot)$ is the trace of a matrix. Therefore, the 2D Barakat degree of polarization, $m_{\mathrm{CP}}=\sqrt{1-\frac{4\left|\mathbf{C}_{2}\right|}{\left(\operatorname{Tr}\left(\mathbf{C}_{2}\right)\right)^{2}}}$ is also roll-invariant. Hence, we conclude that the proposed scattering-type parameter for CP SAR,

$$
\theta_{\mathrm{CP}}=2 \tan ^{-1}\left(\frac{m_{\mathrm{CP}} S_{0}(\mathrm{OC}-\mathrm{SC})}{\mathrm{OC} \times \mathrm{SC}+m_{\mathrm{CP}}^{2} S_{0}^{2}}\right)
$$

is independent of $\Psi$, i.e., it is a roll-invariant parameter. 
560

561

$$
\vec{k}_{\theta, \chi}=\left[\begin{array}{l}
\cos (\chi)\left(\cos (\theta) S_{H H}+\sin (\theta) S_{H V}\right)+i \sin (\chi)\left(\sin (\theta) S_{H H}-\cos (\theta) S_{H V}\right) \\
\cos (\chi)\left(\cos (\theta) S_{V H}+\sin (\theta) S_{V V}\right)+i \sin (\chi)\left(\sin (\theta) S_{V H}-\cos (\theta) S_{V V}\right)
\end{array}\right]
$$

564

$$
\mathbf{U}_{\theta_{r}, \chi_{r}}=\left[\begin{array}{cc}
\cos \left(\theta_{r}\right) \cos \left(\chi_{r}\right)+i \sin \left(\theta_{r}\right) \sin \left(\chi_{r}\right) & \sin \left(\theta_{r}\right) \cos \left(\chi_{r}\right)-i \cos \left(\theta_{r}\right) \sin \left(\chi_{r}\right) \\
-\sin \left(\theta_{r}\right) \cos \left(\chi_{r}\right)-i \cos \left(\theta_{r}\right) \sin \left(\chi_{r}\right) & \cos \left(\theta_{r}\right) \cos \left(\chi_{r}\right)-i \sin \left(\theta_{r}\right) \sin \left(\chi_{r}\right)
\end{array}\right]
$$

${ }_{568}$ The $2 \times 2$ covariance matrix for GTGR becomes,

$$
\mathbf{C} p\left(\theta_{r}, \chi_{r} \mid \theta, \chi\right)=\mathbf{U}_{\theta_{r}, \chi_{r}} \mathbf{C} p(\theta, \chi) \mathbf{U}_{\theta_{r}, \chi_{r}}^{\dagger}
$$


569

where,

$$
\mathbf{C} p(\theta, \chi)=\left\langle\vec{k}_{\theta, \chi} \vec{k}_{\theta, \chi}^{\dagger}\right\rangle
$$

570 571

$$
\gamma_{H}=\frac{\left\langle S_{H H} S_{H V}^{*}\right\rangle}{\sqrt{\left\langle\left|S_{H H}\right|^{2}\right\rangle} \sqrt{\left\langle\left|S_{H V}\right|^{2}\right\rangle}} ; \gamma_{V}=\frac{\left\langle S_{V V} S_{H V}^{*}\right\rangle}{\sqrt{\left\langle\left|S_{V V}\right|^{2}\right\rangle} \sqrt{\left\langle\left|S_{H V}\right|^{2}\right\rangle}} ; \gamma_{H V}=\frac{\left\langle S_{H H} S_{V V}^{*}\right\rangle}{\sqrt{\left\langle\left|S_{H H}\right|^{2}\right\rangle} \sqrt{\left\langle\left|S_{V V}\right|^{2}\right\rangle}}
$$

572 along with the ratio factors,

$$
\mathrm{a}=\sqrt{\frac{\left\langle\left|S_{H V}\right|^{2}\right\rangle}{\left\langle\left|S_{H H}\right|^{2}\right\rangle}}=\sqrt{\frac{\left\langle\sigma_{H V}^{0}\right\rangle}{\left\langle\sigma_{H H}^{0}\right\rangle}} ; b=\sqrt{\frac{\left\langle\left|S_{H V}\right|^{2}\right\rangle}{\left\langle\left|S_{V V}\right|^{2}\right\rangle}}=\sqrt{\frac{\left\langle\sigma_{H V}^{0}\right\rangle}{\left\langle\sigma_{V V}^{0}\right\rangle}} ; c=\frac{a}{b}=\sqrt{\frac{\left\langle\left|S_{V V}\right|^{2}\right\rangle}{\left\langle\left|S_{H H}\right|^{2}\right\rangle}}=\sqrt{\frac{\left\langle\sigma_{V V}^{0}\right\rangle}{\left\langle\sigma_{H H}^{0}\right\rangle}}
$$

where $\sigma^{0}$ represents the normalized radar cross section. Using (B.6), (B.7) and (B.8), the elements of the $2 \times 2$ covariance matrix $\mathbf{C} p(\theta, \chi)$ for the General Transmit and Linear Received (GTLR) can be expressed by,

$$
\begin{gathered}
C p_{11}(\theta, \chi)=\frac{1}{2}\left\langle\left|S_{H H}\right|^{2}\right\rangle\left\{(1+\cos (2 \theta) \cos (2 \chi))+(1-\cos (2 \theta) \cos (2 \chi)) a^{2}+\right. \\
\left.\left.\sin (2 \theta) \cos (2 \chi) a\left(\gamma_{H}+\gamma_{H}^{*}\right)+i \sin (2 \chi) a\left(\gamma_{H}-\gamma_{H}^{*}\right)\right\} \quad \text { B.9 }\right) \\
C p_{12}(\theta, \chi)=C p_{21}^{*}(\theta, \chi)=\frac{1}{2}\left\langle\left|S_{H H}\right|^{2}\right\rangle\left\{(1+\cos (2 \theta) \cos (2 \chi)) a \gamma_{H}+\right. \\
(1-\cos (2 \theta) \cos (2 \chi)) a c \gamma_{V}^{*}+\sin (2 \theta) \cos (2 \chi)\left(c \gamma_{H V}+a^{2}\right)+ \\
\left.\left.i \sin (2 \chi)\left(c \gamma_{H V}-a^{2}\right)\right\} \quad \text { (B. } 10\right)
\end{gathered}
$$




$$
\begin{aligned}
C p_{22}(\theta, \chi)= & \frac{1}{2}\left\langle\left|S_{H H}\right|^{2}\right\rangle\left\{(1+\cos (2 \theta) \cos (2 \chi)) a^{2}+(1-\cos (2 \theta) \cos (2 \chi)) c^{2}+\right. \\
& \left.\left.\sin (2 \theta) \cos (2 \chi) a c\left(\gamma_{V}+\gamma_{V}^{*}\right)-i \sin (2 \chi) a c\left(\gamma_{V}-\gamma_{V}^{*}\right)\right\} \quad \text { B. } 11\right)
\end{aligned}
$$

Therefore, for right circular polarized transmit wave, we have considered, $\chi=-45^{\circ}$ and $\theta=0^{\circ}$. In this work, we have used the European Space Agency's (ESA) open-source toolbox for polarimetric SAR data processing and education PolSARpro (Polarimetric SAR Data Processing and Education Toolbox). We have used this toolbox for simulating CP data from FP SAR data.

\section{Appendix C. Software/Codes to extract FP and CP parameters}

We obtain the $3 \times 3$ coherency matrix, $\mathbf{T}$ from the full-polarimetric SAR data using the PolSARpro software. The compact polarimetric data is simulated using the same software by assuming right-hand circular polarized transmit wave (i.e., $\chi=-45^{\circ}$ ), where $\chi$ is the ellipticity parameter of the polarization ellipse.

All the parameters used in this study (i.e., $m_{\mathrm{FP}}, m_{\mathrm{CP}}, \theta_{\mathrm{FP}}, \theta_{\mathrm{CP}}, H_{\mathrm{FP}}$, and $\left.H_{\mathrm{CP}}\right)$ are computed using scripts developed in MatLaB R2019b environment as shown in figure C.17. For the full-polarimetry data, we read the 9 elements (i.e., 3 positive real diagonal elements and 3 complex off-diagonal elements) of the $\mathbf{T}$ matrix while for compact-polarimetry data, we read the 4 elements (i.e., 2 positive real diagonal elements and 1 complex off-diagonal element) of the $\mathbf{C}_{2}$ matrix. Thereafter, using array solution and iteration methods, we compute the spatial distribution of these parameters. The codes are available at: http://github.com/Subho07/Temporal-clustering-of-SAR-data/ 


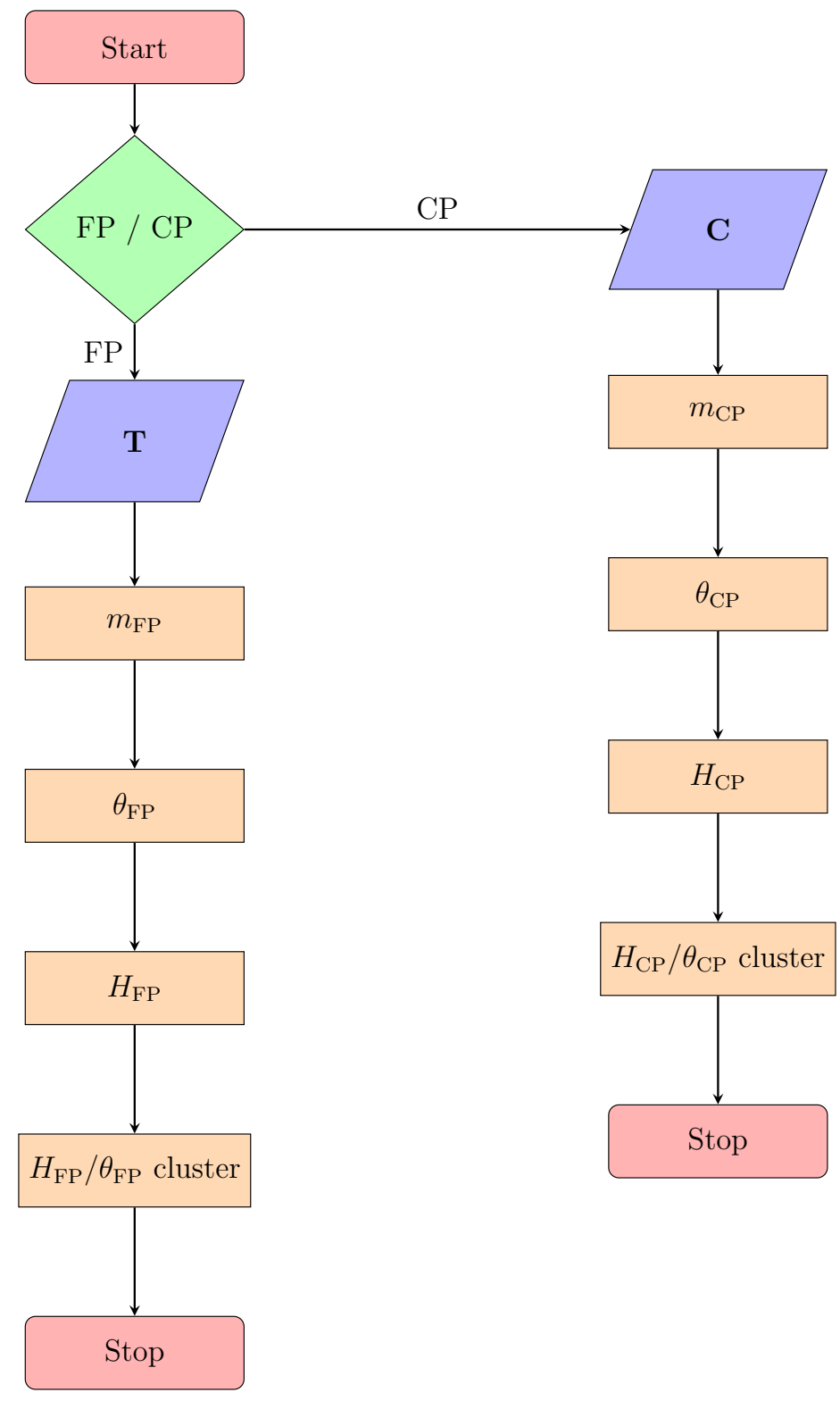

Figure C.17: Flow chart for computing the Barakat degree of polarization $\left(m_{\mathrm{FP}}, m_{\mathrm{CP}}\right)$, target characterizing parameters $\left(\theta_{\mathrm{FP}}, \theta_{\mathrm{CP}}\right)$ and scattering entropy $\left(H_{\mathrm{FP}}, H_{\mathrm{CP}}\right)$ for $\mathrm{FP}$ and CP data using MatLaB R2019b environment. 


\section{Acknowledgements}

The authors want to thank Prof. Alejandro C. Frery, School of Mathematics and Statistics, Victoria University of Wellington, New Zealand, for helping us with the statistical analysis of the clustering scheme. The authors would like to thank the Canadian Space Agency and MAXAR Technologies Ltd. (formerly MDA) for providing RADARSAT-2 images through the Joint Experiment for Crop Assessment and Monitoring (JECAM) Network. The authors are also thankful to Andhra Pradesh Space Application Centre (APSAC), ITE \& C Department, Government of Andhra Pradesh for their support during field campaigns. This work was supported in part by the Spanish Ministry of Science, Innovation and Universities, the State Agency of Research (AEI), and the European Funds for Regional Development (EFRD) under Project TEC 2017-85244-C 2-1-P. The work of Dipankar Mandal was supported by the Ministry of Human Resource Development, Government of India (New Delhi, India) towards his Ph.D. assistantship through grant no. RSPHD0210.

\section{References}

Ainsworth, T., Kelly, J., Lee, J.-S., 2009. Classification comparisons between dual-pol, compact polarimetric and quad-pol SAR imagery. ISPRS Journal of Photogrammetry and Remote Sensing 64 (5), 464-471.

Antropov, O., Rauste, Y., Hame, T., 2011. Volume scattering modeling in PolSAR decompositions: Study of ALOS PALSAR data over boreal forest. IEEE Transactions on Geoscience and Remote Sensing 49 (10), 3838-3848. 
Ballester-Berman, J. D., Lopez-Sanchez, J. M., 2011. Time series of hybridpolarity parameters over agricultural crops. IEEE Geoscience and Remote Sensing Letters 9 (1), 139-143.

Barakat, R., 1977. Degree of polarization and the principal idempotents of the coherency matrix. Optics Communications 23 (2), 147-150.

Barakat, R., 1983. n-fold polarization measures and associated thermodynamic entropy of $\mathrm{N}$ partially coherent pencils of radiation. Optica Acta: International Journal of Optics 30 (8), 1171-1182.

Brisco, B., Li, K., Tedford, B., Charbonneau, F., Yun, S., Murnaghan, K., 2013. Compact polarimetry assessment for rice and wetland mapping. International journal of remote sensing 34 (6), 1949-1964.

Charbonneau, F., Brisco, B., Raney, R., McNairn, H., Liu, C., Vachon, P., Shang, J., DeAbreu, R., Champagne, C., Merzouki, A., et al., 2010. Compact polarimetry overview and applications assessment. Canadian Journal of Remote Sensing 36 (sup2), S298-S315.

Cloude, S. R., Goodenough, D. G., Chen, H., 2011. Compact decomposition theory. IEEE Geoscience and Remote Sensing Letters 9 (1), 28-32.

Cloude, S. R., Pottier, E., 1997. An entropy based classification scheme for land applications of polarimetric SAR. IEEE transactions on geoscience and remote sensing 35 (1), 68-78.

Davidson, M. W., Le Toan, T., Mattia, F., Satalino, G., Manninen, T., Borgeaud, M., 2000. On the characterization of agricultural soil roughness 
for radar remote sensing studies. IEEE Transactions on Geoscience and Remote Sensing 38 (2), 630-640.

De Bernardis, C. G., Vicente-Guijalba, F., Martinez-Marin, T., LopezSanchez, J. M., 2015. Estimation of key dates and stages in rice crops using dual-polarization SAR time series and a particle filtering approach. IEEE Journal of Selected Topics in Applied Earth Observations and Remote Sensing 8 (3), 1008-1018.

Dey, S., Bhattacharya, A., Ratha, D., Mandal, D., Frery, A. C., 2020. Target characterization and scattering power decomposition for full and compact polarimetric SAR data. IEEE Transactions on Geoscience and Remote Sensing.

Guo, X., Li, K., Shao, Y., Wang, Z., Li, H., Yang, Z., Liu, L., Wang, S., 2018. Inversion of rice biophysical parameters using simulated compact polarimetric SAR C-band data. Sensors 18 (7), 2271.

He, Z., Li, S., Wang, Y., Dai, L., Lin, S., 2018. Monitoring rice phenology based on backscattering characteristics of multi-temporal RADARSAT-2 datasets. Remote Sensing 10 (2), 340.

Kumar, V., Mandal, D., Bhattacharya, A., Rao, Y., 2020. Crop characterization using an improved scattering power decomposition technique for compact polarimetric sar data. International Journal of Applied Earth Observation and Geoinformation 88, 102052.

Lee, J.-S., Pottier, E., 2009. Polarimetric radar imaging: from basics to applications. CRC press. 
Lopez-Sanchez, J. M., Ballester-Berman, J. D., Hajnsek, I., 2011. First results of rice monitoring practices in spain by means of time series of TerraSAR-X dual-pol images. IEEE Journal of selected topics in applied earth observations and remote sensing 4 (2), 412-422.

Lopez-Sanchez, J. M., Cloude, S. R., Ballester-Berman, J. D., 2012. Rice phenology monitoring by means of SAR polarimetry at X-band. IEEE Transactions on Geoscience and Remote Sensing 50 (7), 2695-2709.

Lopez-Sanchez, J. M., Vicente-Guijalba, F., Ballester-Berman, J. D., Cloude, S. R., 2014. Polarimetric response of rice fields at C-band: Analysis and phenology retrieval. IEEE Transactions on Geoscience and Remote Sensing $52(5), 2977-2993$.

Mandal, D., Kumar, V., Rao, Y., Bhattacharya, A., Ramana, K., 2019. Experimental field campaigns at Vijayawada test site. Tech. Rep. MRS2019TR02, Microwave Remote Sensing Lab, India.

URL http://doi.org/10.17605/OSF. IO/DN3E8

Mandal, D., Kumar, V., Ratha, D., Lopez-Sanchez, J. M., Bhattacharya, A., McNairn, H., Rao, Y., Ramana, K., 2020. Assessment of rice growth conditions in a semi-arid region of india using the generalized radar vegetation index derived from radarsat-2 polarimetric sar data. Remote Sensing of Environment 237, 111561.

McNairn, H., Jiao, X., Pacheco, A., Sinha, A., Tan, W., Li, Y., 2018. Estimating canola phenology using synthetic aperture radar. Remote sensing of environment 219, 196-205. 
McNairn, H., Shang, J., 2016. A review of multitemporal synthetic aperture radar (SAR) for crop monitoring. In: Multitemporal Remote Sensing. Springer, pp. 317-340.

Paloscia, S., 2002. A summary of experimental results to assess the contribution of SAR for mapping vegetation biomass and soil moisture. Canadian Journal of Remote Sensing 28 (2), 246-261.

Praks, J., Koeniguer, E. C., Hallikainen, M. T., 2009. Alternatives to target entropy and alpha angle in SAR polarimetry. IEEE Transactions on Geoscience and Remote Sensing 47 (7), 2262-2274.

Raney, R. K., 2007. Hybrid-polarity SAR architecture. IEEE Transactions on Geoscience and Remote Sensing 45 (11), 3397-3404.

Raney, R. K., Cahill, J. T., Patterson, G. W., Bussey, D. B. J., 2012. The mchi decomposition of hybrid dual-polarimetric radar data with application to lunar craters. Journal of Geophysical Research: Planets 117 (E12).

Ratha, D., Pottier, E., Bhattacharya, A., Frery, A. C., 2019. A PolSAR scattering power factorization framework and novel roll-invariant parameterbased unsupervised classification scheme using a geodesic distance. IEEE Transactions on Geoscience and Remote Sensing, 1-17.

Sabry, R., Vachon, P. W., 2013. A unified framework for general compact and quad polarimetric SAR data and imagery analysis. IEEE Transactions on Geoscience and Remote Sensing 52 (1), 582-602.

Touzi, R., Hurley, J., Vachon, P. W., 2015. Optimization of the degree of 
polarization for enhanced ship detection using polarimetric RADARSAT-2. IEEE Transactions on Geoscience and Remote Sensing 53 (10), 5403-5424.

Touzi, R., Omari, K., Sleep, B., Jiao, X., 2018. Scattered and received wave polarization optimization for enhanced peatland classification and fire damage assessment using polarimetric PALSAR. IEEE Journal of Selected Topics in Applied Earth Observations and Remote Sensing 11 (11), $4452-4477$.

Uppala, D., Kothapalli, R. V., Poloju, S., Mullapudi, S. S. V. R., Dadhwal, V. K., 2015. Rice crop discrimination using single date RISAT1 hybrid (RH, RV) polarimetric data. Photogrammetric Engineering \& Remote Sensing 81 (7), 557-563.

Wiseman, G., McNairn, H., Homayouni, S., Shang, J., 2014. RADARSAT2 polarimetric SAR response to crop biomass for agricultural production monitoring. IEEE Journal of Selected Topics in Applied Earth Observations and Remote Sensing 7 (11), 4461-4471.

Xie, L., Zhang, H., Wu, F., Wang, C., Zhang, B., 2015. Capability of rice mapping using hybrid polarimetric SAR data. IEEE Journal of Selected Topics in Applied Earth Observations and Remote Sensing 8 (8), 38123822.

Yang, Z., Li, K., Liu, L., Shao, Y., Brisco, B., Li, W., 2014. Rice growth monitoring using simulated compact polarimetric C band SAR. Radio Science 49 (12), 1300-1315. 
729 Yin, J., Moon, W. M., Yang, J., 2015. Novel model-based method for identi730 fication of scattering mechanisms in polarimetric SAR data. IEEE Trans${ }_{731}$ actions on Geoscience and Remote Sensing 54 (1), 520-532.

732 Yin, J., Papathanassiou, K. P., Yang, J., 2019. Formalism of compact po733 larimetric descriptors and extension of the $\triangle \alpha B / \alpha B$ method for general 734 compact-pol SAR. IEEE Transactions on Geoscience and Remote Sensing, $735 \quad 1-14$.

736 Yonezawa, C., Negishi, M., Azuma, K., Watanabe, M., Ishitsuka, N., Ogawa, ${ }_{737}$ S., Saito, G., 2012. Growth monitoring and classification of rice fields using multitemporal RADARSAT-2 full-polarimetric data. International journal of remote sensing 33 (18), 5696-5711.

Yuzugullu, O., Erten, E., Hajnsek, I., 2015. Rice growth monitoring by means of X-band co-polar SAR: Feature clustering and bbch scale. IEEE Geoscience and Remote Sensing Letters 12 (6), 1218-1222. 